\title{
Evaluación de leguminosas como abono verde en cultivos forrajeros para ganaderías en el Caribe seco colombiano ${ }^{1}$
}

\section{Evaluation of legumes as green manure in forage crops for livestock in the dry Colombian Caribbean}

\author{
Edwin Castro-Rincón ${ }^{2}$, José Edwin Mojica-Rodríguez ${ }^{3}$, Juan Evangelista Carulla-Fornaguera ${ }^{4}$, \\ Carlos Eduardo Lascano-Aguilar ${ }^{5}$
}

\begin{abstract}
Resumen
Los sistemas ganaderos de doble propósito en el trópico son afectados durante la época seca con reducción en la producción de leche, debido a la disminución en la cantidad y calidad de los forrajes. Se puede disminuir el impacto mediante el uso de cultivos forrajeros como suplementos generados con el uso de abonos verdes. El objetivo de esta investigación fue determinar el aporte de nitrógeno de leguminosas en la producción de forraje de maíz. Durante los años 2013 a 2014, en el Caribe Seco Colombiano, se establecieron tres experimentos con siete niveles de nitrógeno (N) $(0,25,50,75,100,150$ y $200 \mathrm{~kg} / \mathrm{ha})$ y la incorporación de Canavalia brasiliensis (CIAT 17009), Vigna unguiculata (CIAT 4294), Clitoria ternatea (CIAT 21815), Canavalia ensiformis (CIAT 21826) y Lablab purpureus (CIAT 22656), en un diseño de bloques al azar. C. ensiformis presentó el mayor aporte de N (151 kg/ha) y V. unguiculata (35 kg/ha) el menor. La aplicación de $200 \mathrm{~kg}$ de $\mathrm{N}$ y la incorporación de C. brasiliensis y C. ensiformis no mostró diferencias en el rendimiento de biomasa de maíz. La eficiencia de uso de $\mathrm{N}$ del maíz fue del 31-84\%, reducida con aumento en la dosis de $\mathrm{N}$. Los niveles de $\mathrm{NO}_{3}$ en el suelo variaron entre 20 y $41 \mathrm{mg} / \mathrm{kg}$ al momento de la cosecha, sugiriendo que una proporción del $\mathrm{N}$ aportado por la leguminosa no fue asimilado por el cultivo de maíz. La relación C:N fue 11,63 en las leguminosas y 23,94 en las arvenses. El carbono orgánico en el suelo aumentó en 22\% con abonos verdes, los mayores contenidos $(0,70 \%)$ fueron obtenidos con L. purpureus. Las leguminosas C. ensiformis, C. brasiliensis y L. purpureus mostraron alto potencial para aportar N, C y conservar humedad del suelo.
\end{abstract}

Palabras clave: fertilidad del suelo, maíz, carbono, plantas de cobertura.

\begin{abstract}
The dual-purpose livestock systems in the tropics milk production is reduced during the dry season due to the decrease in the quantity and quality of forage on offer. The impact can be reduced by using forage crops as supplements generated with the use of green fertilizers. The objective of this research was to determinate the nitrogen
\end{abstract}

1 Recibido: 8 de febrero, 2018. Aceptado: 14 de mayo, 2018. Este trabajo formó parte del trabajo de tesis doctoral del primer autor: "Utilización de leguminosas forrajeras como abonos verdes para la producción de cultivos forrajeros y leche en ganaderías doble propósito en el trópico seco", en la Universidad Nacional de Colombia, sede Bogotá. Financiado por la Corporación Colombiana de Investigación Agropecuaria (AGROSAVIA). Colombia.

2 Corporación Colombiana de Investigación Agropecuaria (AGROSAVIA). Nariño, Colombia. ecastro@agrosavia.co (autor para correspondencia).

3 Corporación Colombiana de Investigación Agropecuaria (AGROSAVIA). Agustín Codazzi, César, Colombia. jemojica@agrosavia.co

4 Universidad Nacional de Colombia, Facultad de Medicina Veterinaria y de Zootecnia. Bogotá, Colombia. jecarullaf@unal.edu.co

5 Emérito Centro Internacional de Agricultura Tropical (CIAT).Cali, Colombia. c.lascano@cgiar.org 
contribution of legumes in corn forage production. During the years 2013 to 2014, in the Colombian Dry Caribbean three experiments were established with seven levels of nitrogen $(\mathrm{N})(0,25,50,75,100,150$ and $200 \mathrm{~kg} / \mathrm{ha})$ and the incorporation of Canavalia brasiliensis (CIAT 17009), Vigna unguiculata (CIAT 4294), Clitoria ternatea (CIAT 21815), Canavalia ensiformis (CIAT 21826) and Lablab purpureus (CIAT 22656) in a random block design. $C$. ensiformis showed the highest contribution of $\mathrm{N}(151 \mathrm{~kg} / \mathrm{ha})$ and $V$. unguiculata the lowest $(35 \mathrm{~kg} / \mathrm{ha})$. The application of $200 \mathrm{~kg}$ of $\mathrm{N}$ and the incorporation of $C$. ensiformis and C. brasiliensis did not show a difference in the yield of maize biomass. $\mathrm{N}$ use efficiency by corn crop was $31-84 \%$, reduced with increased in the dose of $\mathrm{N}$ Levels of $\mathrm{NO}_{3}$ in the soil ranged from 20 to $41 \mathrm{mg} / \mathrm{kg}$ at the time of harvest, suggesting that a high proportion of $\mathrm{N}$ coming from the legume was not assimilated by the crop. The C:N ratio was 11.63 in legumes and 23.94 in weeds. Organic soil $\mathrm{C}$ increased by $22 \%$ due to legume green manure, with the highest content $(0.70 \%)$ in L.purpureus. The legumes $C$. ensiformis, C. brasiliensis and L. purpureus showed high potential to provide N, C, and to conserve soil moisture.

Keywords: soil fertility, corn, carbon, cover plants.

\section{Introducción}

En la actualidad se realiza un uso intensivo de la tierra, con efectos adversos en la fertilidad del suelo, agotamiento de nutrientes y erosión (Tan et al., 2005). En el trópico seco hay una disminución drástica de la producción agrícola y pecuaria asociada a los fuertes periodos secos y procesos avanzados de degradación de suelos (Douxchamps et al., 2010). A fines de suplir las deficiencias en producción de biomasa para alimentación de animales, se opta por la producción de cultivos forrajeros como maíz, especie dependiente de la aplicación de nitrógeno químico para obtener rendimientos adecuados (Chikowo et al., 2006). Sin embargo, en la mayoría de los casos no se aplican cantidades adecuadas de fertilizantes y en las explotaciones agrícolas se presenta el agotamiento de $\mathrm{N}$ que se traduce en una importante limitante para la producción (Smyth et al., 2004; Ayarza et al., 2007). Como estrategia de suministro de $\mathrm{N}$, surge el uso de leguminosas como abonos verdes, tecnología ya documentada en diferentes escenarios, tanto para cultivos de maíz como de arroz (Burle et al., 1992; Biederbeck et al., 1998; Mureithi et al., 2003; Castro, 2016; Castro et al., 2017), donde con la incorporación de las leguminosas como fuente de nutrientes al suelo, se ha logrado reemplazar parte de la fertilización inorgánica, mejorando las propiedades físicas y químicas del suelo (Douxchamps et al., 2014; Castro et al., 2016).

El uso de leguminosas herbáceas como abonos verdes es una opción para productores de leche en sistemas doble propósito en el trópico bajo, dada su capacidad de fijar $\mathrm{N}$ en el suelo y la posibilidad de integrarlas en sistemas de rotación con cultivos forrajeros (Castro, 2016).

En Latinoamérica se han evaluado distintas especies de leguminosas como abonos verdes en diferentes sistemas de producción, las cuales han manifestado aportes de $\mathrm{N}$ entre 60 y $300 \mathrm{~kg} / \mathrm{ha}$ (Thurston, 1992; Cherr et al., 2006). También, experimentos en Guatemala mostraron que el valor de sustitución de fertilizante nitrogenado con Mucuna spp. y C. ensiformis incorporados fue entre 60 y $158 \mathrm{~kg} \mathrm{~N} / \mathrm{ha}$ (Pound, 1999).

En Nicaragua se evaluó $C$. brasiliensis y su aporte de $\mathrm{N}$ como abono verde, la producción de biomasa de la leguminosa varió entre 2448 y $5357 \mathrm{~kg} / \mathrm{ha}$, con una tasa de incorporación de N entre 150-200 kg/ha (Douxchamps, 2010). En investigaciones realizadas en el este de Uganda se observaron rendimientos de hasta un 50 a $60 \%$ más altos del maíz cuando se usaron Crotalaria ochroleuca, M. pruriens, D. lablab y C. ensiformis como abonos verdes, en comparación a cuando no se utilizaron (Mureithi et al., 2003). En Brasil se realizaron ensayos en arroz con el uso de $\mathrm{N}$ químico (urea) y abonos verdes de $C$. juncea y $M$. aterrina, observándose niveles de sustitución de hasta 40 $\mathrm{kg} / \mathrm{ha}$ de $\mathrm{N}$ con el uso de leguminosas como fuente de nitrógeno (Muraoka et al., 2002; Carvalho et al., 2004). En 
Colombia se han evaluado como abonos verdes, leguminosas como C. cajan y C. ensiformis en el Valle del Cauca (Salamanca et al., 2004), C. retusa, C. juncea, C. ensiformis y V. unguiculata en los llanos orientales (Navas y Bernal, 1999), C. brasiliensis y V. unguiculata en el Valle del César (Castro, 2016; Castro et al., 2017), en todos los casos hubo buena producción de biomasa y nutrientes para el suelo y el cultivo posterior, siendo estas leguminosas potenciales para uso como abono verde en la producción de cultivos forrajeros utilizados en la ganadería.

Para definir el potencial de uso de abonos verdes en sistemas ganaderos en el trópico, se realizó el presente estudio con el objetivo de determinar el aporte equivalente de $\mathrm{N}$ de leguminosas empleadas como abono verde en la producción de forraje de maíz.

\section{Materiales y métodos}

La investigación se realizó en el Centro de Investigación (CI) Motilona de la Corporación Colombiana de Investigación Agropecuaria (AGROSAVIA), ubicado en Agustín Codazzi, y en dos fincas localizadas en los municipios de La Paz (Doña Rosa) y Valledupar (Salguero), microrregión Valle del César, con condiciones de suelo caracterizadas al inicio de los experimentos (Cuadro 1), que reflejaron adecuadas condiciones de fertilidad en términos de $\mathrm{pH}$ y disponibilidad de macronutrientes. Se ubicaron tres sitios para replicar los experimentos tanto en el manejo dentro de un centro de investigación, como en fincas en condiciones contrastantes de la zona.

Cuadro 1. Análisis de suelos de los sitios donde se realizaron los experimentos de evaluación de niveles de $\mathrm{N}$ y abonos verdes en Valle del César, César, Colombia. 2013-2014.

Table 1. Soil analysis of the sites where the evaluation experiments of $\mathrm{N}$ levels and green fertilizers were carried out in Valle del Cesar, Cesar, Colombia. 2013-2014.

\begin{tabular}{|c|c|c|c|c|c|c|c|c|c|}
\hline \multirow[t]{2}{*}{ Finca } & \multirow[t]{2}{*}{$\mathrm{pH}^{1}$} & \multirow[t]{2}{*}{ Textura $^{2}$} & \multirow[t]{2}{*}{ MO \% } & \multirow{2}{*}{$\begin{array}{c}P \\
\mathrm{mg} / \mathrm{kg}\end{array}$} & \multirow[t]{2}{*}{$\mathbf{S}$} & \multirow{2}{*}{$\begin{array}{c}\mathrm{Ca} \\
\mathrm{cmol} / \mathrm{kg}\end{array}$} & \multirow[t]{2}{*}{ Mg } & \multirow[t]{2}{*}{$\mathbf{K}$} & \multirow[t]{2}{*}{$\mathrm{Na}$} \\
\hline & & & & & & & & & \\
\hline Valledupar & 7,2 & FraA & 1,2 & 419,66 & 3,1 & 4,89 & 2,08 & 2,06 & 0,09 \\
\hline La Paz & 7,5 & Fra & 1,1 & 25,97 & 2,9 & 4,19 & 0,62 & 0,30 & 0,06 \\
\hline Agustín Codazzi & 6,6 & FraA & 1,9 & 512,99 & 4,2 & 6,40 & 0,89 & 0,51 & 0,05 \\
\hline
\end{tabular}

${ }^{1}$ Métodos según el laboratorio de análisis de suelos de AGROSAVIA. ${ }^{2}$ FraA: franco arcillo arenosa, Fra: franco arcilloso $/{ }^{1}$ Methods according to the laboratory of soil analysis of AGROSAVIA. ${ }^{2}$ FraA: frank sandy clay, Fra: clay loam.

Se evaluaron en total doce tratamientos, siete niveles de fertilización nitrogenada y cinco leguminosas incorporadas al suelo como abono verde, como fuentes de incorporación de N. Los tratamientos fueron: T1: 0 kg N/ha, T2: $25 \mathrm{~kg} \mathrm{~N} / \mathrm{ha}$, T3: $50 \mathrm{~kg} \mathrm{~N} / \mathrm{ha}$, T4: $75 \mathrm{~kg} \mathrm{~N} / \mathrm{ha}$, T5: $100 \mathrm{~kg} \mathrm{~N} / \mathrm{ha}$, T6: $150 \mathrm{~kg} \mathrm{~N} / \mathrm{ha}, \mathrm{T} 7: 200 \mathrm{~kg} \mathrm{~N} / \mathrm{ha}$, T8: incorporación de C. brasiliensis (CIAT 17009), T9: incorporación de Vigna unguiculata (CIAT 4294), T10: incorporación de Clitoria ternatea (CIAT 21815), T11: incorporación de Canavalia ensiformis (CIAT 21826), T12: Incorporación de Lablab purpureus (CIAT 22656).

Las leguminosas se establecieron en los tres sitios mencionados al final del periodo de lluvias del primer semestre de 2013, y se dejaron sembradas durante toda la época seca, para ser incorporadas al suelo al inicio del siguiente periodo de lluvias. Cada parcela o unidad experimental recibió una fertilización de establecimiento para garantizar que no existiera limitación de nutrientes (P: 25, K: 20, Mg: 10, S: 10, B: $10 \mathrm{~kg} / \mathrm{ha}$ ). El tamaño de cada parcela fue de $20 \mathrm{~m}^{2}(4 \mathrm{~m} \times 5 \mathrm{~m})$, con una separación de $1 \mathrm{~m}$ entre calles y $2 \mathrm{~m}$ entre repeticiones. La parcela 
estuvo conformada por nueve surcos de $5 \mathrm{~m}$ de longitud, separados a $50 \mathrm{~cm}$ entre sí; la distancia entre plantas en cada surco fue de $50 \mathrm{~cm}$ entre sitios.

A comienzos del periodo de lluvias del segundo semestre de 2013, luego de 85 días de siembra, se incorporaron las leguminosas al suelo, cortándolas, dejándolas secar e incorporando con la preparación del suelo, luego a los quince días se sembró maíz (variedad- ICA-V109) como cultivo indicador. Cada parcela estuvo conformada por seis surcos de $5 \mathrm{~m}$ de largo cada uno, separados a $70 \mathrm{~cm}$ y con cinco a siete plantas de maíz por metro lineal. Las dosis de $\mathrm{N}$ se aplicaron en forma fraccionada en tres aplicaciones a los 10, 20 y 32 días después de emergencia de las plantas de maíz.

\section{Variables medidas}

En cada sitio se determinó la producción de materia seca de la leguminosa (Toledo y Shultze-Kraft, 1982) y el maíz (Boschini y Elizondo, 2004), además, el contenido de nutrientes (N, P, Ca, Mg y K) de la biomasa de las leguminosas incorporadas y del cultivo indicador (maíz). En el suelo se determinó el contenido de $\mathrm{NO}_{3}$ (Phiri et al., 2001), N total (Krom, 1980) y carbono total (C) (Nelson y Sommers, 1982). El N equivalente aportado por cada leguminosa se calculó por medio de una ecuación de regresión lineal $(\mathrm{Y}=\mathrm{a}+\mathrm{bX})$, donde $\mathrm{Y}$ fue el rendimiento de maíz y X la cantidad de N químico aplicado al maíz en los ensayos de niveles de nitrógeno (a: intercepto, b: pendiente).

\section{Diseño experimental y análisis estadístico}

Se empleó un diseño de bloques completos al azar, con siete niveles de $\mathrm{N}$, cinco leguminosas y tres repeticiones en cada localidad, para un total de 36 parcelas experimentales por sitio. Los resultados se analizaron usando el PROC GLM del paquete estadístico SAS (V 9.3), y para la comparación de medias se usó la prueba Tukey.

\section{Resultados}

\section{Producción de biomasa y aporte de $\mathbf{N}$ en leguminosas}

En el experimento localizado en el Centro de Investigación Motilonia (Agustín Codazzi), los resultados mostraron diferencias $(\mathrm{p}<0,001)$ en la producción de biomasa entre las leguminosas (Cuadro 2). La mayor producción se observó con C. ensiformis ( $6428 \mathrm{~kg}$ Ms/ha) y la menor con C. ternatea (2651 kg MS/ha). En el sitio de la Paz también la de mayor producción fue C. ensiformis $(\mathrm{p}<0,05)$ frente a C. ternatea (6589 y $2779 \mathrm{~kg} \mathrm{MS} / \mathrm{ha})$ (Cuadro 2). En el sitio de Valledupar se obtuvo la mayor producción con C. ensiformis (6186 kg MS/ha) y la menor $(\mathrm{p}<0,05)$ con $V$. unguiculata $(2643 \mathrm{~kg} \mathrm{MS} / \mathrm{ha})$.

El equivalente de $\mathrm{N}$ ( $\mathrm{kg} \mathrm{N} / \mathrm{ha}$ ) aportado por las leguminosas incorporadas al suelo se cuantificó con una ecuación de regresión ( $\mathrm{Y}=13681,5+20,3 \mathrm{X} ; \mathrm{r}=0,84)$, obtenida a partir de la producción de biomasa de maíz (Y) y las dosis de $\mathrm{N}$ aplicadas (X). Tanto en el CI Motilonia como en La Paz y Valledupar, el mayor equivalente de N se observó con C. ensiformis (151, 166 y $173 \mathrm{~kg} \mathrm{~N} / \mathrm{ha}$, respectivamente). El menor equivalente en el CI Motilonia y en Valledupar se observó en V. unguiculata (35 y $86 \mathrm{~kg} \mathrm{~N} / \mathrm{ha}$, respectivamente) (Cuadro 3). Mientras en La Paz el menor equivalente se obtuvo con C. ternatea ( $90 \mathrm{~kg} \mathrm{~N} / \mathrm{ha}$ ) (Cuadro 2). El valor promedio de equivalentes de $\mathrm{N}$ fue mayor en La Paz, asociado a mayores niveles de producción de biomasa de maíz como respuesta a los diferentes niveles de $\mathrm{N}$ aplicados y como respuesta en la planta. 
Cuadro 2. Producción de biomasa y aporte de N equivalente por cuatro leguminosas evaluadas como abono verde en tres localidades. César, Colombia. 2013-2014.

Table 2. Biomass production and $\mathrm{N}$ equivalent contribution by four legumes evaluated as green manure in three locations. Cesar, Colombia. 2013-2014.

\begin{tabular}{lcccccc}
\hline \multirow{2}{*}{ Leguminosa } & \multicolumn{2}{c}{ Agustín Codazzi } & \multicolumn{2}{c}{ La Paz } & \multicolumn{2}{c}{ Valledupar } \\
\cline { 2 - 7 } & ${ }^{1} \mathbf{k g ~ M S} / \mathbf{h a}$ & ${ }^{1} \mathbf{k g N} / \mathbf{h a}$ & $\mathbf{k g ~ M S} / \mathbf{h a}$ & $\mathbf{k g N} / \mathbf{h a}$ & $\mathbf{k g ~ M S / h a}$ & $\mathbf{k g N} / \mathbf{h a}$ \\
\hline C. ternatea & $2651 \mathrm{~d}$ & $106 \mathrm{ab}$ & $2779 \mathrm{~b}$ & $90 \mathrm{~b}$ & $2776 \mathrm{~d}$ & $106 \mathrm{~b}$ \\
V. unguiculata & $3891 \mathrm{c}$ & $35 \mathrm{bc}$ & $3193 \mathrm{~b}$ & $126 \mathrm{~b}$ & $2643 \mathrm{~d}$ & $86 \mathrm{~b}$ \\
C. ensiformis & $6428 \mathrm{a}$ & $151 \mathrm{a}$ & $6589 \mathrm{a}$ & $166 \mathrm{a}$ & $6186 \mathrm{a}$ & $173 \mathrm{a}$ \\
L. purpureus & $5565 \mathrm{ab}$ & $103 \mathrm{ab}$ & $6382 \mathrm{a}$ & $171 \mathrm{a}$ & $4924 \mathrm{c}$ & $159 \mathrm{a}$ \\
C. brasiliensis & $5076 \mathrm{~b}$ & $129 \mathrm{a}$ & $5276 \mathrm{a}$ & $115 \mathrm{~b}$ & $5513 \mathrm{~b}$ & $109 \mathrm{~b}$ \\
Biomasa no leguminosa & $1824 \mathrm{~d}$ & $-37 \mathrm{c}$ & $2102 \mathrm{~b}$ & $-7 \mathrm{c}$ & $1810 \mathrm{e}$ & $-19 \mathrm{c}$ \\
\hline Promedio & 4239 & 81,2 & 4386 & 110 & 3976 & 102 \\
\hline
\end{tabular}

Medias seguidas por letras iguales en la misma columna no son significativamente diferentes ( $<<0,05)$, según prueba de Tukey. ${ }^{1}$ Calculado con la ecuación: X: (Y - a) / b derivada de la ecuación de regresión: Y: 13681,48 + 20,30X; r: 0,84: donde Y: kg MS/ha de maíz y X kg de N/ha aplicado / Means followed by the equal letters in the same column are not significantly different (p<0.05), according to Tukey's test. ${ }^{1}$ Calculated with the equation: X: (Y - a) / b derived from the regression equation: Y: 13681.48 + 20.30X; r: 0.84: where Y: $\mathrm{kg} \mathrm{MS/ha}$ of corn and $\mathrm{X} \mathrm{kg}$ of N/ha applied.

\section{Contenido de minerales en las leguminosas}

Los resultados obtenidos en el Centro de Investigación Motilona en Agustín Codazzi no mostraron diferencias ( $p>0,05$ ) en el contenido de K entre leguminosas. Sin embargo, los contenidos de N, P, Ca y Mg fueron diferentes $(\mathrm{p}<0,05)$ entre leguminosas (Cuadro 3). El contenido de $\mathrm{N}$ varió entre 3,1 y 3,5\% en las leguminosas y 1,5\% en el maíz (testigo negativo). El nivel de $\mathrm{P}$ en las leguminosas evaluadas varió entre 0,2 y 0,5\%; mientras que, los niveles de Ca variaron entre 1,3 y $4,3 \%$ y los de $\mathrm{S}$ entre 0,2 y $0,8 \%$.

Cuadro 3. Contenido de minerales (\%) en la parte aérea de las leguminosas evaluadas como abonos verdes en Agustín Codazzi, César, Colombia. 2013-2014.

Table 3. Mineral content (\%) in the aerial part of the legumes evaluated as green manures in Agustin Codazzi, Cesar, Colombia. 2013-2014.

\begin{tabular}{lllllll}
\hline Tratamiento & $\mathbf{N}$ & $\mathbf{P}$ & $\mathbf{C a}$ & $\mathbf{M g}$ & $\mathbf{K}$ & $\mathbf{S}$ \\
\hline C. ternatea & $3,5^{1}$ & $0,45 \mathrm{ab}^{1}$ & $1,25 \mathrm{~cd}^{1}$ & $0,47 \mathrm{~b}^{1}$ & $2,44^{1}$ & $0,23 \mathrm{bc}^{1}$ \\
V. unguiculata & 3,4 & $0,46 \mathrm{ab}$ & $4,29 \mathrm{a}$ & $0,51 \mathrm{~b}$ & 3,84 & $0,24 \mathrm{bc}$ \\
C. ensiformis & 3,3 & $0,20 \mathrm{c}$ & $2,45 \mathrm{bc}$ & $0,21 \mathrm{~b}$ & 1,91 & $0,48 \mathrm{~b}$ \\
L. purpureus & 3,1 & $0,54 \mathrm{a}$ & $2,59 \mathrm{~b}$ & $0,27 \mathrm{~b}$ & 3,72 & $0,20 \mathrm{c}$ \\
C. brasiliensis & 3,3 & $0,36 \mathrm{~b}$ & $4,08 \mathrm{a}$ & $0,27 \mathrm{~b}$ & 2,22 & $0,17 \mathrm{c}$ \\
No leguminosa & 1,5 & $0,37 \mathrm{~b}$ & $0,36 \mathrm{~d}$ & $2,82 \mathrm{a}$ & 1,60 & $0,77 \mathrm{a}$ \\
\hline Promedio & 3,03 & 0,40 & 2,50 & 0,76 & 2,62 & 0,35 \\
\hline
\end{tabular}

${ }^{1}$ Medias seguidas por letras iguales en la misma columna no son significativamente diferentes $(\mathrm{p}<0,05)$, según prueba de Tukey / Means followed by the equal letter in the same column are not significantly different $(\mathrm{p}<0.05)$, according to Tukey's test. 
En La Paz se observaron diferencias en el contenido de Ca, K y S $(\mathrm{p}<0,05)$ (Cuadro 4) entre leguminosas, no siendo así para $\mathrm{N}$ y P, donde las diferencias observadas fueron hacia el maíz.

Cuadro 4. Contenido de minerales (\%) en la parte aérea de las leguminosas evaluadas como abonos verdes en La Paz, César, Colombia. 2013-2014.

Table 4. Mineral content (\%) in the aerial part of the legumes evaluated as green manures in La Paz, Cesar, Colombia. $2013-2014$.

\begin{tabular}{lcccccc}
\hline Tratamiento & $\mathbf{N}$ & $\mathbf{P}$ & $\mathbf{C a}$ & $\mathbf{M g}$ & $\mathbf{K}$ & $\mathbf{S}$ \\
\hline C. ternatea & $3,03^{1}$ & $0,35^{1}$ & $1,42^{1} \mathrm{ab}$ & $0,46^{1}$ & $2,78^{1} \mathrm{ab}$ & $2,60^{1} \mathrm{ab}$ \\
V. unguiculata & 2,89 & 0,27 & $2,34 \mathrm{a}$ & 0,32 & $1,80 \mathrm{~b}$ & $2,46 \mathrm{ab}$ \\
C. ensiformis & 3,09 & 0,21 & $2,06 \mathrm{a}$ & 0,45 & $1,99 \mathrm{~b}$ & $2,87 \mathrm{a}$ \\
L. purpureus & 3,11 & 0,21 & $2,16 \mathrm{a}$ & 0,35 & $2,02 \mathrm{~b}$ & $1,45 \mathrm{ab}$ \\
C. brasiliensis & 3,15 & 0,34 & $1,56 \mathrm{ab}$ & 0,35 & $3,15 \mathrm{a}$ & $2,50 \mathrm{ab}$ \\
No leguminosa & 1,18 & 0,23 & $0,87 \mathrm{~b}$ & 0,39 & $2,75 \mathrm{ab}$ & $0,83 \mathrm{~b}$ \\
\hline Promedio & & 0,27 & 1,74 & 0,39 & 2,41 & \\
\hline
\end{tabular}

${ }^{1}$ Medias seguidas por letras iguales en la misma columna no son significativamente diferentes $(\mathrm{p}<0,05)$, según prueba de Tukey / Means followed by the equal letters in the same column are not significantly different $(\mathrm{p}<0.05)$, according to Tukey's test.

En Valledupar se observaron diferencias para todos los minerales $(\mathrm{p}<0,05)$ (Cuadro 5). Pero en general, dentro de las tres localidades no se observaron diferencias mayores entre los contenidos de las leguminosas evaluadas $(\mathrm{p}>0,05)$.

Cuadro 5. Contenido de minerales (\%) en la parte aérea de las leguminosas evaluadas como abonos verdes en Valledupar, César, Colombia. 2013-2014.

Table 5. Mineral content (\%) in the aerial part of the legumes evaluated as green manures in Valledupar, Cesar, Colombia. 20132014.

\begin{tabular}{lcccccc}
\hline Tratamiento & $\mathbf{N}$ & $\mathbf{P}$ & $\mathbf{C a}$ & $\mathbf{M g}$ & $\mathbf{K}$ & $\mathbf{S}$ \\
\hline C. ternatea & $3,08^{1} \mathrm{ab}$ & $0,21^{1} \mathrm{ab}$ & $1,14^{1} \mathrm{~b}$ & $0,39^{1} \mathrm{ab}$ & $1,78^{1} \mathrm{~b}$ & $2,88^{1} \mathrm{a}$ \\
V. unguiculata & $2,87 \mathrm{~b}$ & $0,29 \mathrm{a}$ & $1,64 \mathrm{ab}$ & $0,47 \mathrm{a}$ & $1,76 \mathrm{~b}$ & $1,45 \mathrm{ab}$ \\
C. ensiformis & $3,08 \mathrm{ab}$ & $0,19 \mathrm{~b}$ & $1,70 \mathrm{ab}$ & $0,47 \mathrm{a}$ & $2,39 \mathrm{ab}$ & $0,77 \mathrm{~b}$ \\
L. purpureus & $3,48 \mathrm{a}$ & $0,17 \mathrm{~b}$ & $1,99 \mathrm{a}$ & $0,17 \mathrm{~b}$ & $2,95 \mathrm{a}$ & $2,43 \mathrm{a}$ \\
C. brasiliensis & $2,65 \mathrm{~b}$ & $0,14 \mathrm{~b}$ & $1,87 \mathrm{a}$ & $0,33 \mathrm{ab}$ & $1,80 \mathrm{~b}$ & $2,19 \mathrm{ab}$ \\
No leguminosa & $1,12 \mathrm{c}$ & $0,18 \mathrm{~b}$ & $1,05 \mathrm{~b}$ & $0,36 \mathrm{ab}$ & $2,86 \mathrm{a}$ & $2,04 \mathrm{ab}$ \\
\hline Promedio & 2,71 & 0,19 & 1,73 & 0,38 & 2,41 & 2,12 \\
\hline
\end{tabular}

Medias seguidas por letras iguales en la misma columna no son significativamente diferentes $(\mathrm{p}<0,05)$, según prueba de Tukey / Means followed by the equal letters in the same column are not significantly different $(\mathrm{p}<0.05)$, according to Tukey's test.

\section{Producción de biomasa en cultivo indicador (maíz)}

En Agustín Codazzi, con la incorporación de C. brasiliensis, C. ensiformis, C. ternatea y L. purpureus, la producción de forraje de maíz no fue diferente $(\mathrm{p}<0,05)$ a la producción que se obtuvo con $200 \mathrm{~kg}$ de N/ha; pero con esta última dosis, la producción de forraje de maíz fue superior en un $34 \%$ a la que se obtuvo cuando no se aplicó N. 
Con la incorporación de C. ensiformis al suelo (equivalente a $151 \mathrm{~kg} \mathrm{~N} / \mathrm{ha}$ ), se logró una producción de forraje de maíz $20 \%$ superior a la que se obtuvo con la dosis comúnmente usada en la zona ( $50 \mathrm{~kg} \mathrm{~N} / \mathrm{ha}$ ), y $4 \%$ inferior a la producción que se obtuvo con la dosis más alta de N (200 kg/ha) aplicado (Cuadro 6).

Cuadro 6. Producción de biomasa del cultivo indicador (maíz), aporte de N y eficiencia de uso de N (EFN) en el maíz en los diferentes tratamientos en Agustín Codazzi, César, Colombia. 2013-2014.

Table 6. Biomass production of indicator crop (maize), N contribution and efficiency of use of N (EFN) in corn in the different treatments in Agustin Codazzi, Cesar, Colombia. 2013-2014.

\begin{tabular}{|c|c|c|c|}
\hline Tratamiento & kg MS/ha & kg N/ha en maíz ${ }^{1}$ & Eficiencia uso $\mathbf{N}$ \\
\hline $0 \mathrm{~N}$ & $12933 \mathrm{e}$ & $157,7 \mathrm{~d}$ & \\
\hline $25 \mathrm{~N}$ & $14765 \mathrm{~cd}$ & $171,2 \mathrm{~cd}$ & $61,4 \mathrm{ab}$ \\
\hline $50 \mathrm{~N}$ & 13993 de & $181,9 \mathrm{bc}$ & $48,5 \mathrm{bc}$ \\
\hline $75 \mathrm{~N}$ & $16089 a b c$ & $202,2 \mathrm{ab}$ & $59,4 \mathrm{~b}$ \\
\hline $100 \mathrm{~N}$ & $16275 \mathrm{ab}$ & $205,8 \mathrm{ab}$ & $48,1 \mathrm{bc}$ \\
\hline $150 \mathrm{~N}$ & $16475 \mathrm{ab}$ & $204,7 \mathrm{ab}$ & $31,4 \mathrm{c}$ \\
\hline $200 \mathrm{~N}$ & $17420 \mathrm{a}$ & $222,7 \mathrm{a}$ & $32,5 \mathrm{c}$ \\
\hline C. brasiliensis & $16300 \mathrm{ab}$ & $224,1 \mathrm{a}$ & $51,3 \mathrm{bc}$ \\
\hline C. ensiformis & $16743 \mathrm{ab}$ & 225,9 a & $45,1 \mathrm{bc}$ \\
\hline V. unguiculata & $14388 \mathrm{~d}$ & $185,6 \mathrm{bc}$ & 83,6 a \\
\hline C.ternatea & $15821 \mathrm{bc}$ & $205,8 \mathrm{ab}$ & $49,4 \mathrm{bc}$ \\
\hline L.purpureus & $15757 \mathrm{bc}$ & $194,6 \mathrm{bc}$ & $39,2 \mathrm{bc}$ \\
\hline Promedio & 15580 & 198,6 & 50,0 \\
\hline
\end{tabular}

Medias seguidas por letras iguales en la misma columna no son significativamente diferentes ( $<<0,05)$, según prueba de Tukey. ${ }^{1}$ Calculado por la producción de biomasa de maíz: $\mathrm{kg} \mathrm{MS} / \mathrm{ha} * \% \mathrm{~N}$ maíz / Means followed by the equal letters in the same column are not significantly different $(\mathrm{p}<0.05)$, according to Tukey's test. ${ }^{1}$ Calculated by the production of corn biomass: $\mathrm{kg} \mathrm{MS} / \mathrm{ha} * \% \mathrm{~N}$ corn.

Para La Paz, se logró la mayor producción de forraje de maíz con la dosis de $150 \mathrm{~kg}$ de $\mathrm{kg} \mathrm{N} / \mathrm{ha}$, la cual no fue diferente de la incorporación de C. ensiformis ni a la dosis más alta de $\mathrm{N}(\mathrm{p}<0,05)$ (Cuadro 7).

En Valledupar la mayor producción se obtuvo al incorporar C. ensiformis $(14797 \mathrm{~kg} \mathrm{MS} / \mathrm{ha})(\mathrm{p}<0,05)$, pero sin diferencias a lo obtenido con 150 y $200 \mathrm{~kg} \mathrm{~N} / \mathrm{ha}$ (Cuadro 8).

El contenido de $\mathrm{N}$ en el maíz para el sitio de Agustín Codazzi fue mayor con la incorporación de C. ensiformis y C. brasiliensis $(225,9$ y $224,1 \mathrm{~kg} \mathrm{~N} / \mathrm{ha})$, mientras el menor contenido fue para el tratamiento de $0 \mathrm{~N}(\mathrm{p}<0,05)$ (157,7 kg N/ha) (Cuadro 7). En la Paz se observó con L. purpureus (242,2 kg N/ha) el contenido más alto de N en el maíz cosechado (Cuadro 7).

En Valledupar se obtuvo el mayor contenido de $\mathrm{N}$ en la cosecha del cultivo indicador con el tratamiento con $C$. ensiformis (214 kg N/ha) (Cuadro 8). En las tres localidades el menor contenido de $\mathrm{N}$ en el maíz se obtuvo con 0N.

En las tres localidades la eficiencia aparente de uso del $\mathrm{N}$ por parte del cultivo de maíz, calculada por método indirecto ( $\mathrm{kg} \mathrm{N} / \mathrm{ha}$ planta fertilizada - $\mathrm{kg} \mathrm{N} / \mathrm{ha}$ planta sin fertilizar)/kg N/ha aplicado o incorporado), se redujo a medida que aumentó la dosis de N, siendo en todos los casos la más baja con la dosis de $200 \mathrm{~kg}$ N/ha (Figura 1). En otros estudios también se ha encontrado una menor eficiencia en uso de $\mathrm{N}$ a medida que se aumenta la dosis sobre 125 - $150 \mathrm{~kg} \mathrm{~N} / \mathrm{ha}$ (Soto et al., 2004; Valero et al., 2005; Barbieri et al., 2010) (Cuadros 7, 8 y 9). 
Cuadro 7. Producción de biomasa del cultivo indicador (maíz), aporte de $\mathrm{N}$ y eficiencia de uso de $\mathrm{N}$ (EUN) en el maíz en los diferentes tratamientos en la Paz, César, Colombia. 2013-2014.

Table 7. Biomass production of indicator crop (maize), $\mathrm{N}$ contribution and efficiency of use of $\mathrm{N}$ (EFN) in corn in the different treatments in La Paz, Cesar, Colombia. 2013-2014.

\begin{tabular}{|c|c|c|c|}
\hline Tratamiento & Kg MS/ha & Kg N/ha en maíz ${ }^{1}$ & Eficiencia uso $\mathbf{N}$ \\
\hline $\mathrm{ON}$ & $11669 \mathrm{~g}$ & $135,6 \mathrm{f}$ & \\
\hline $25 \mathrm{~N}$ & $11627 \mathrm{~g}$ & $146,3 \mathrm{ef}$ & $41,2 \mathrm{ab}$ \\
\hline $50 \mathrm{~N}$ & $13775 \mathrm{f}$ & 159,4 def & $46,9 \mathrm{ab}$ \\
\hline $75 \mathrm{~N}$ & $13760 \mathrm{f}$ & $158,3 \mathrm{def}$ & $29,7 \mathrm{~b}$ \\
\hline $100 \mathrm{~N}$ & $15825 \mathrm{bcd}$ & $190,4 \mathrm{bcd}$ & $54,4 \mathrm{ab}$ \\
\hline $150 \mathrm{~N}$ & 17049 a & $210,4 \mathrm{abc}$ & $49,6 \mathrm{ab}$ \\
\hline $200 N$ & $16222 \mathrm{abcd}$ & 183,2 cde & $23,6 \mathrm{~b}$ \\
\hline C. brasiliensis & $15106 \mathrm{de}$ & $180,5 \mathrm{cde}$ & $38,4 \mathrm{ab}$ \\
\hline C. ensiformis & $16536 \mathrm{abc}$ & $201,1 \mathrm{bc}$ & $39,1 \mathrm{ab}$ \\
\hline V. unguiculata & 15401 cde & $223,6 \mathrm{ab}$ & $68,4 \mathrm{a}$ \\
\hline C.ternatea & 14406 ef & 175,7 cde & $44,4 a b$ \\
\hline L. purpureus & $16663 a b$ & $242,2 \mathrm{a}$ & $60,9 \mathrm{ab}$ \\
\hline Promedio & 14836 & 183,9 & 45,17 \\
\hline
\end{tabular}

Medias seguidas por letras iguales en la misma columna no son significativamente diferentes ( $\mathrm{p}<0,05$ ), según prueba de Tukey. ${ }^{1}$ Calculado por la producción de biomasa de maíz: $\mathrm{kg} \mathrm{MS} / \mathrm{ha} \% \% \mathrm{~N}$ maíz / Means followed by the equal letters in the same column are not significantly different $(\mathrm{p}<0.05)$, according to Tukey's test. ${ }^{1}$ Calculated by the production of corn biomass: $\mathrm{kg}$ MS/ha $* \% \mathrm{~N}$ corn.

Cuadro 8. Producción de biomasa del cultivo indicador (maíz), aporte de $\mathrm{N}$ y eficiencia de uso de $\mathrm{N}$ (EUN) en el maíz en los diferentes tratamientos en Valledupar, César, Colombia. 2013-2014.

Table 8. Biomass production of indicator crop (maize), $\mathrm{N}$ contribution and efficiency of use of $\mathrm{N}$ (EFN) in corn in the different treatments in Valledupar, Cesar, Colombia. 2013-2014.

\begin{tabular}{|c|c|c|c|}
\hline Tratamiento & kg MS/ha & kg N/ha en maíz ${ }^{1}$ & Eficiencia uso $\mathrm{N}$ \\
\hline $0 \mathrm{~N}$ & $10880 \mathrm{e}$ & $125,4 \mathrm{e}$ & \\
\hline $25 \mathrm{~N}$ & 11547 e & $135,6 \mathrm{de}$ & $42,5 \mathrm{abc}$ \\
\hline $50 \mathrm{~N}$ & $13006 \mathrm{~d}$ & $156,8 \mathrm{~cd}$ & $63,7 \mathrm{a}$ \\
\hline $75 \mathrm{~N}$ & $13693 \mathrm{bcd}$ & $154,7 \mathrm{~cd}$ & $39,6 \mathrm{abc}$ \\
\hline $100 \mathrm{~N}$ & 13828 abcd & $156,9 \mathrm{~cd}$ & $31,9 \mathrm{bc}$ \\
\hline $150 \mathrm{~N}$ & $14222 a b c$ & $169,0 \mathrm{c}$ & $29,3 \mathrm{c}$ \\
\hline $200 N$ & $14641 \mathrm{ab}$ & $177,0 \mathrm{bc}$ & $26,0 \mathrm{c}$ \\
\hline C. brasiliensis & $13486 \mathrm{~cd}$ & $192,5 \mathrm{ab}$ & $60,3 \mathrm{a}$ \\
\hline C. ensiformis & $14797 \mathrm{a}$ & $214 \mathrm{a}$ & $50,8 \mathrm{abc}$ \\
\hline V. unguiculata & $13021 \mathrm{~d}$ & $173,0 \mathrm{bc}$ & $58,0 \mathrm{ab}$ \\
\hline C. ternatea & $13429 \mathrm{~cd}$ & $172,0 \mathrm{bc}$ & $44,6 \mathrm{abc}$ \\
\hline L.purpureus & $14499 \mathrm{ab}$ & 203,8 a & $50,5 \mathrm{abc}$ \\
\hline Promedio & 13421 & 169,2 & 45,2 \\
\hline
\end{tabular}

Medias seguidas por letras iguales en la misma columna no son significativamente diferentes $(\mathrm{p}<0,05)$, según prueba de Tukey. ${ }^{1}$ Calculado por la producción de biomasa de maíz: $\mathrm{kg} \mathrm{MS} / \mathrm{ha} * \% \mathrm{~N}$ maíz / Means followed by the equal letters in the same column are not significantly different $(\mathrm{p}<0.05)$, according to Tukey's test. ${ }^{1}$ Calculated by the production of corn biomass: $\mathrm{kg} \mathrm{MS} / \mathrm{ha} * \% \mathrm{~N}$ corn. 


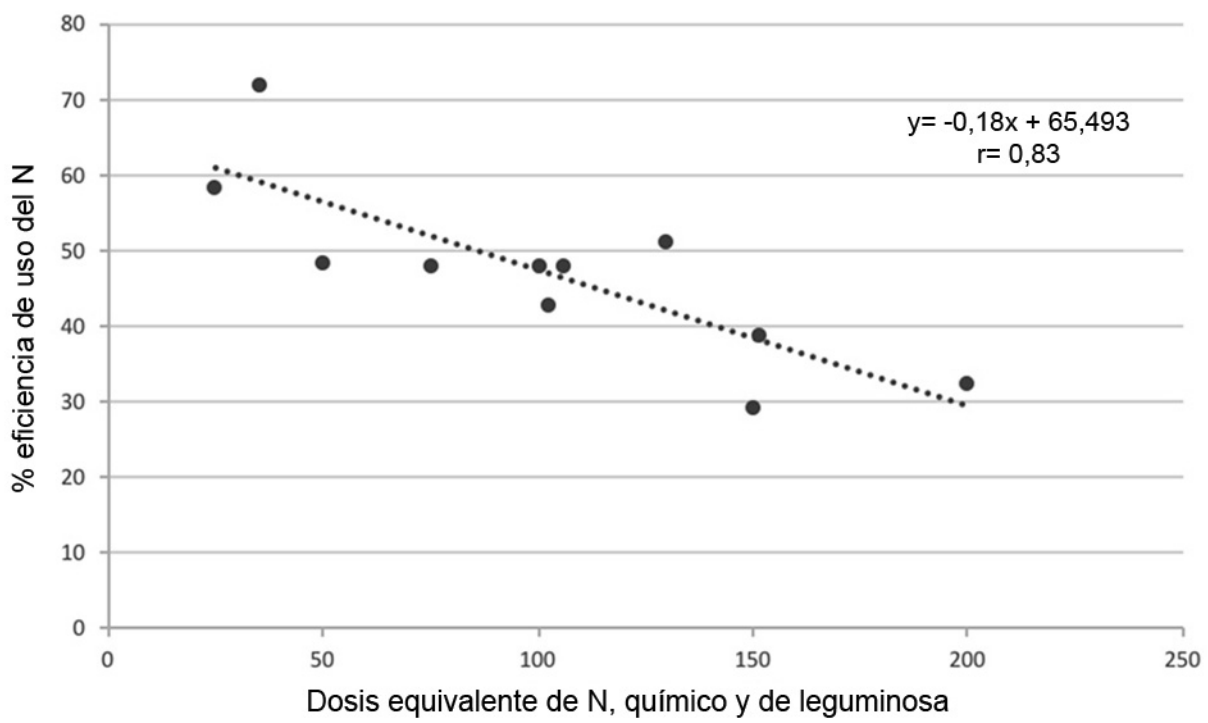

Figura 1. Relación entre nivel de $\mathrm{N}$ incorporado (químico y de abono) y eficiencia aparente de uso del nitrógeno en Valle del César, César, Colombia. 2013-2014.

Figure 1. A relationship between the level of incorporated $\mathrm{N}$ and apparent efficiency of nitrogen use in Valle del Cesar, Cesar, Colombia. 2013-2014.

Cuadro 9. Relación carbono nitrógeno $(\mathrm{C}: \mathrm{N})$ y humedad del suelo en las leguminosas evaluadas en las tres localidades de estudio, Valle del César, César, Colombia. 2013. 2014.

Table 9. Carbon-nitrogen ratio $(\mathrm{C}: \mathrm{N})$ and soil moisture in the legumes evaluated in the three study locations, Valle del Cesar, Cesar, Colombia. 2013. 2014.

\begin{tabular}{lcc}
\hline Leguminosa & Relación C:N en leguminosas & Contenido de humedad en el suelo $(\%)$ \\
\hline C. ternatea & $11,64 \mathrm{~b}$ & $13,48 \mathrm{ab}$ \\
V. unguiculata & $13,12 \mathrm{~b}$ & $11,30 \mathrm{~b}$ \\
C. ensiformis & $12,33 \mathrm{~b}$ & $13,70 \mathrm{ab}$ \\
L. purpureus & $12,96 \mathrm{~b}$ & $14,63 \mathrm{a}$ \\
C. brasiliensis & $11,89 \mathrm{~b}$ & $13,53 \mathrm{ab}$ \\
Biomasa no leguminosa & $22,66 \mathrm{a}$ & $8,96 \mathrm{c}$ \\
\hline Promedio & 14,10 & 12,60 \\
\hline
\end{tabular}

Medias seguidas por letras iguales en la misma columna no son significativamente diferentes $(\mathrm{p}>0,05)$, según prueba de Tukey / Means followed by the equal letters in the same column are not significantly different ( $>0.05)$, according to Tukey's test.

\section{Nitrógeno en el suelo}

En las tres localidades el contenido de $\mathrm{N}$ total en el suelo al momento de la aplicación de $\mathrm{N}$ y de la incorporación de las leguminosas no varió $(\mathrm{p}>0,05)$ por los tratamientos aplicados ni la localidad $(\mathrm{p}<0,05)$, y como era de esperarse fue menor $(\mathrm{p}<0,05)$ en todos los tratamientos al final del ciclo de cosecha del forraje de maíz (Figuras 2 , 
3,4). Se observó además que, al finalizar el ciclo del cultivo indicador (maíz), el contenido de $\mathrm{N}$ total en el suelo tuvo poca variación $(p>0,05)$ entre tratamientos de $\mathrm{N}$ aplicado y de incorporación de leguminosas (Figura 2). En

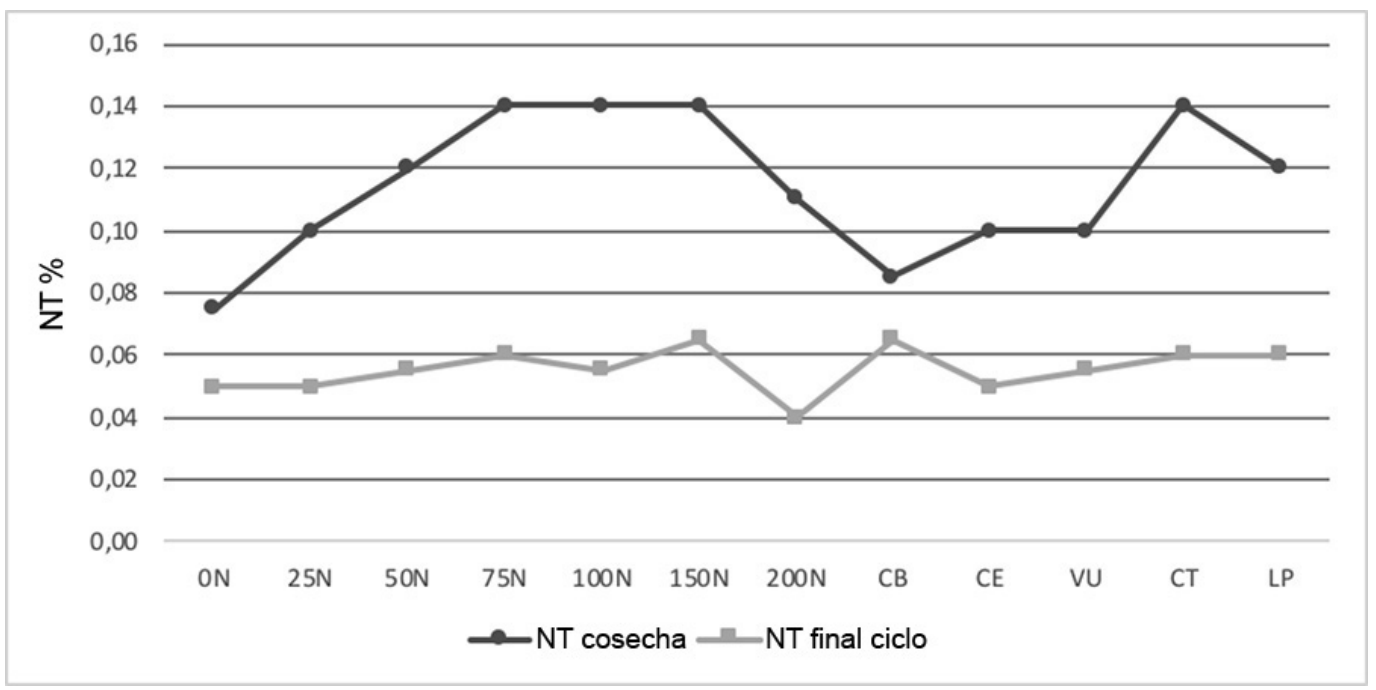

Figura 2. Dinámica de nitrógeno total (NT) en el suelo $(0-20 \mathrm{~cm})$ antes de la incorporación de la leguminosa y al final del ciclo del maíz en Agustín Codazzi, César, Colombia. 2013-2014.

CB: C. brasiliensis; CE: C. ensiformis; VU: V. unguiculata; CT: C. ternatea; VU: V. unguiculata. LP: L. purpureus.

Figure 2. Total nitrogen (NT) dynamics in the soil $(0-20 \mathrm{~cm})$ before the incorporation of the legume and at the end of the corn cycle in Agustin Codazzi, Cesar, Colombia. 2013-2014.

CB: C. brasiliensis; CE: C. ensiformis; VU: V. unguiculata; CT: C. ternatea; VU: V. unguiculata. LP: L. purpureus.

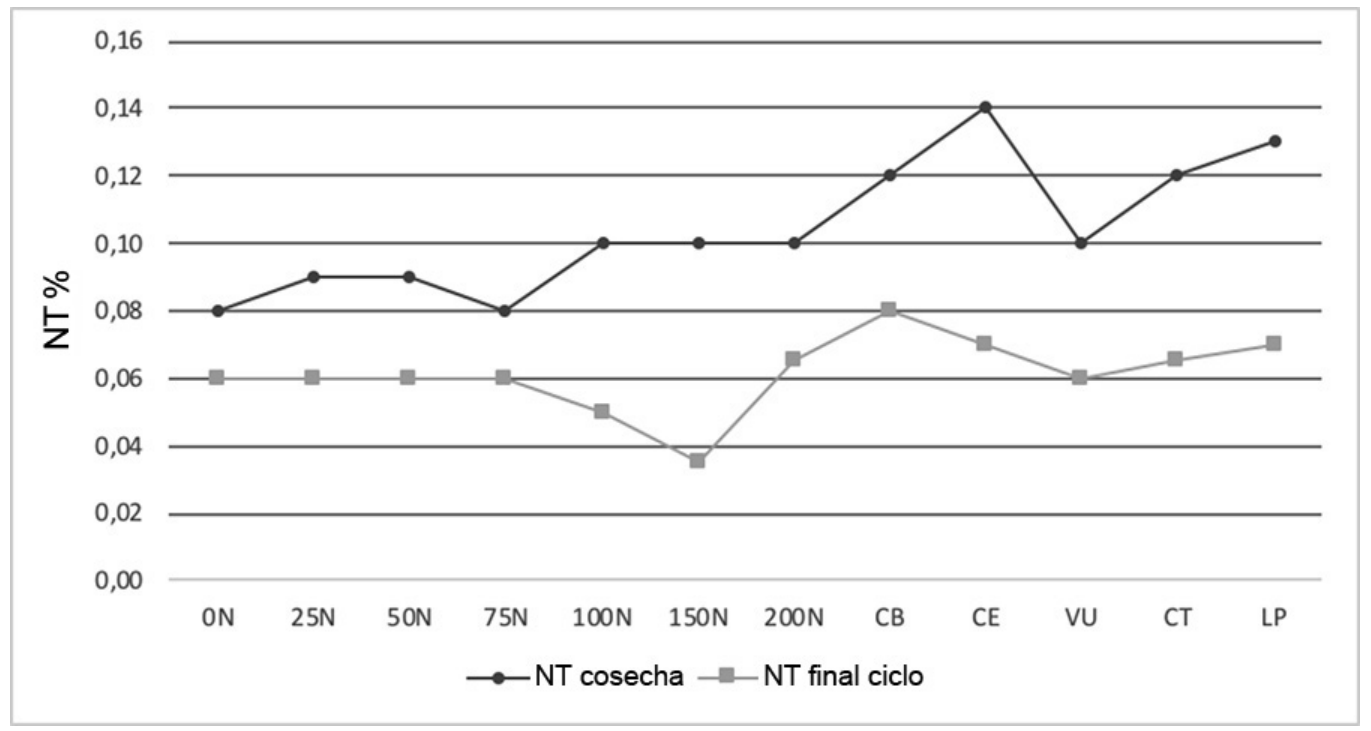

Figura 3. Dinámica de nitrógeno total (NT) en el suelo $(0-20 \mathrm{~cm})$ antes de la incorporación de la leguminosa y al final del ciclo del maíz en la Paz, César, Colombia. 2013-2014.

CB: C. brasiliensis; CE: C. ensiformis; VU: V. unguiculata; CT: C. ternatea; VU: V. unguiculata. LP: L. purpureus..

Figure 3. Total nitrogen (NT) dynamics in the soil $(0-20 \mathrm{~cm})$ before the incorporation of the legume and at the end of the corn cycle in La Paz, Cesar, Colombia. 2013-2014.

CB: C. brasiliensis; CE: C. ensiformis; VU: V. unguiculata; CT: C. ternatea; VU: V. unguiculata. LP: L. purpureus. 


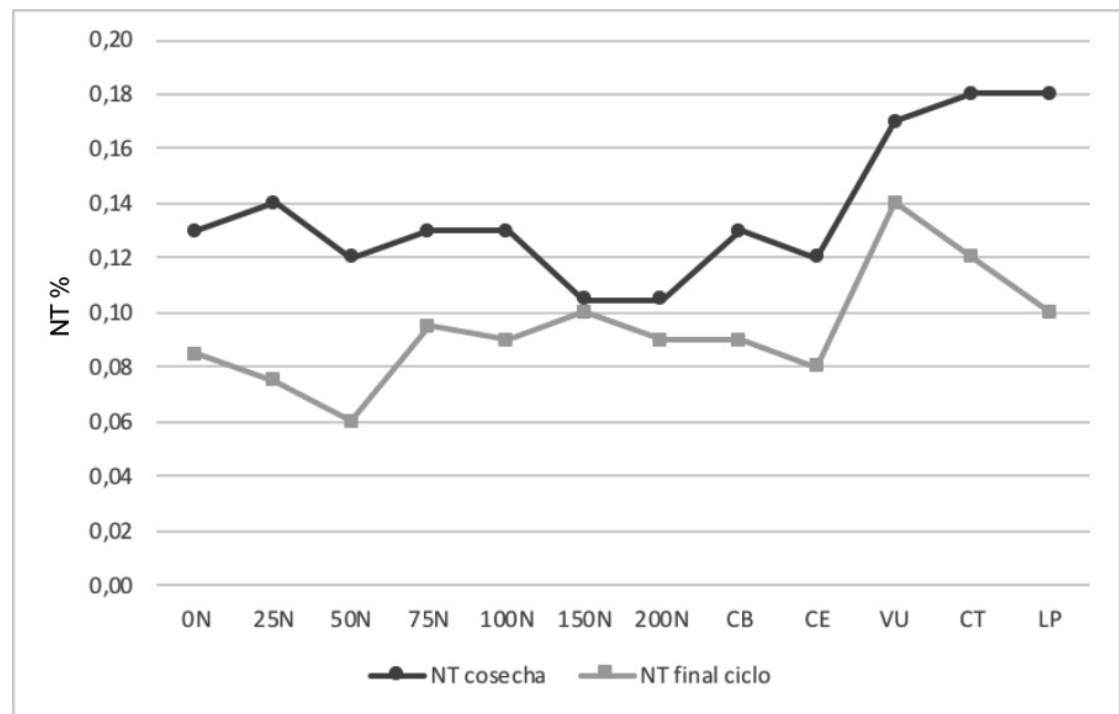

Figura 4. Dinámica de nitrógeno total $(\mathrm{NT})$ en el suelo $(0-20 \mathrm{~cm})$ antes de la incorporación de la leguminosa y al final del ciclo del maíz en Valledupar, César, Colombia. 2013-2014.

CB: C. brasiliensis; CE: C. ensiformis; VU: V. unguiculata; CT: C. ternatea; VU: V. unguiculata. LP: L. purpureus.

Figure 4. Total nitrogen (NT) dynamics in the soil $(0-20 \mathrm{~cm})$ before the incorporation of the legume and at the end of the corn cycle in Valledupar, César, Colombia. 2013-2014.

CB: C. brasiliensis; CE: C. ensiformis; VU: V. unguiculata; CT: C. ternatea; VU: V. unguiculata. LP: L. purpureus.

las localidades de La Paz y Valledupar el nivel de $\mathrm{N}$ total al momento de la incorporación de las leguminosas fue mayor en los tratamientos con leguminosas frente a los de fertilización con $N$ químico $(p<0,05)$.

En el caso de los niveles de nitrato $\left(\mathrm{NO}_{3}\right)$ en el suelo se observó que, en las tres zonas fueron mayores al finalizar el ciclo de maíz que al momento de fertilizar con $\mathrm{N}$ o incorporar leguminosas al suelo. También fue evidente que los valores de $\mathrm{NO}_{3}$ en el suelo fueron muy variables cuando se aplicó $\mathrm{N}$ o se incorporó la leguminosa $(\mathrm{p}<0,05)$. Pero en general, para las tres localidades se observaron niveles altos de $\mathrm{NO}_{3}$ al finalizar el ciclo de cultivo del maíz, con rangos entre 30 y $55 \mathrm{mg} / \mathrm{kg}$ (Figuras $5,6,7$ ).

\section{Relación Carbono:Nitrógeno (C:N) en leguminosas y humedad en suelo}

Dado que no se presentó diferencia para estos parámetros entre las tres localidades $(\mathrm{p}<0,05)$, los datos se muestran como promedio general, con las diferencias de tratamientos en cada caso. La relación C:N más alta $(\mathrm{p}<0,05)(22,66)$ se observó en el material no leguminoso, sin diferencias entre las leguminosas evaluadas $(\mathrm{p}>0,05)$ (Cuadro 8).

Para las tres localidades, el contenido de humedad en el suelo medido antes de la incorporación de las leguminosas fue mayor $(\mathrm{p}<0,05)$ en las parcelas con leguminosa incorporada, con un rango de 11,30 a 13,70\%, frente a las parcelas sin incorporación de abono verde (8,96\%) (Cuadro 9).

El contenido de $\mathrm{C}$ orgánico en el suelo fue superior $(\mathrm{p}<0,05)$ en las tres zonas al momento de cosecha del maíz en comparación con el momento de incorporación de las leguminosas. Se observó un rango que varió desde 0,35\%, en el momento de incorporación de los abonos, hasta 1,4\% al final del ciclo de cosecha del maíz, siendo mayor el contenido en la localidad de Valledupar, en los tratamientos donde se incorporó leguminosa como abono verde $(\mathrm{p}<0,05)$ (Figuras 8, 9, 10). 


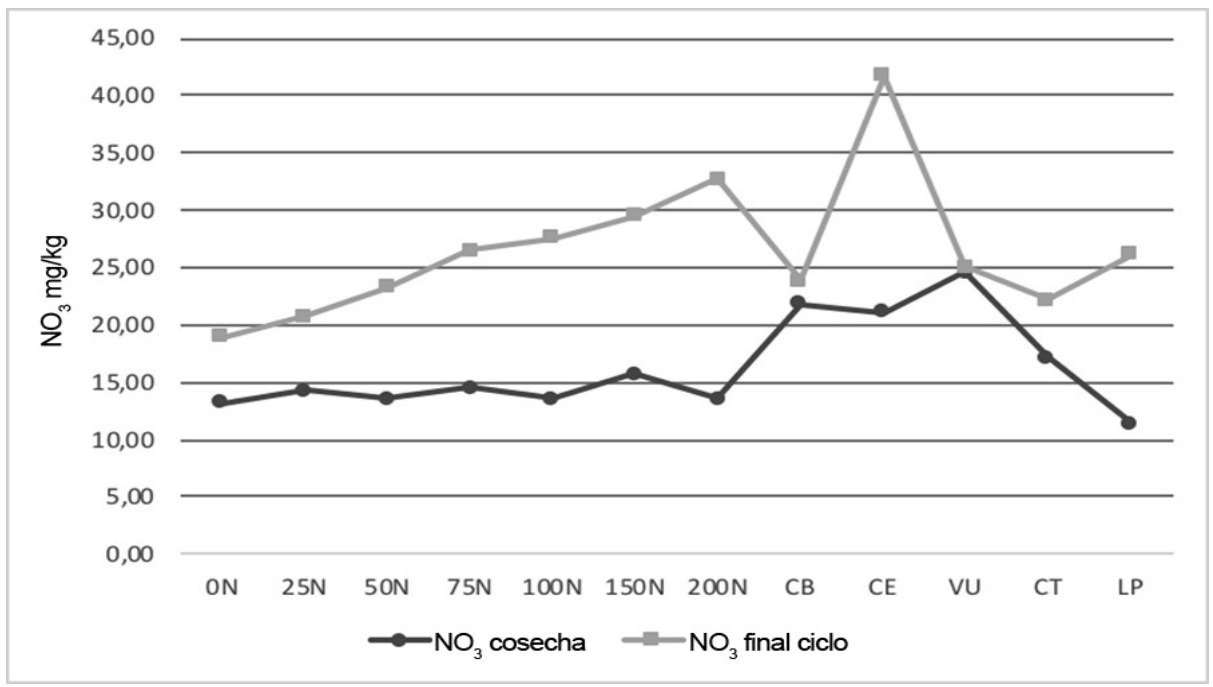

Figura 5. Dinámica de nitrato disponible $(\mathrm{NO} 3)$ en el suelo $(0-20 \mathrm{~cm})$ antes de la incorporación de la leguminosa y al final del ciclo del maíz en Agustín Codazzi, César, Colombia. 2013-2014.

CB: C. brasiliensis; CE: C. ensiformis; VU: V. unguiculata; CT: C. ternatea; VU: V. unguiculata. LP: L. purpureus.

Figure 5. Available nitrate dynamics (NO3) in the soil $(0-20 \mathrm{~cm})$ before the incorporation of the legume and at the end of the corn cycle in Agustin Codazzi, Cesar, Colombia. 2013-2014.

CB: C. brasiliensis; CE: C. ensiformis; VU: V. unguiculata; CT: C. ternatea; VU: V. unguiculata. LP: L. purpureus.

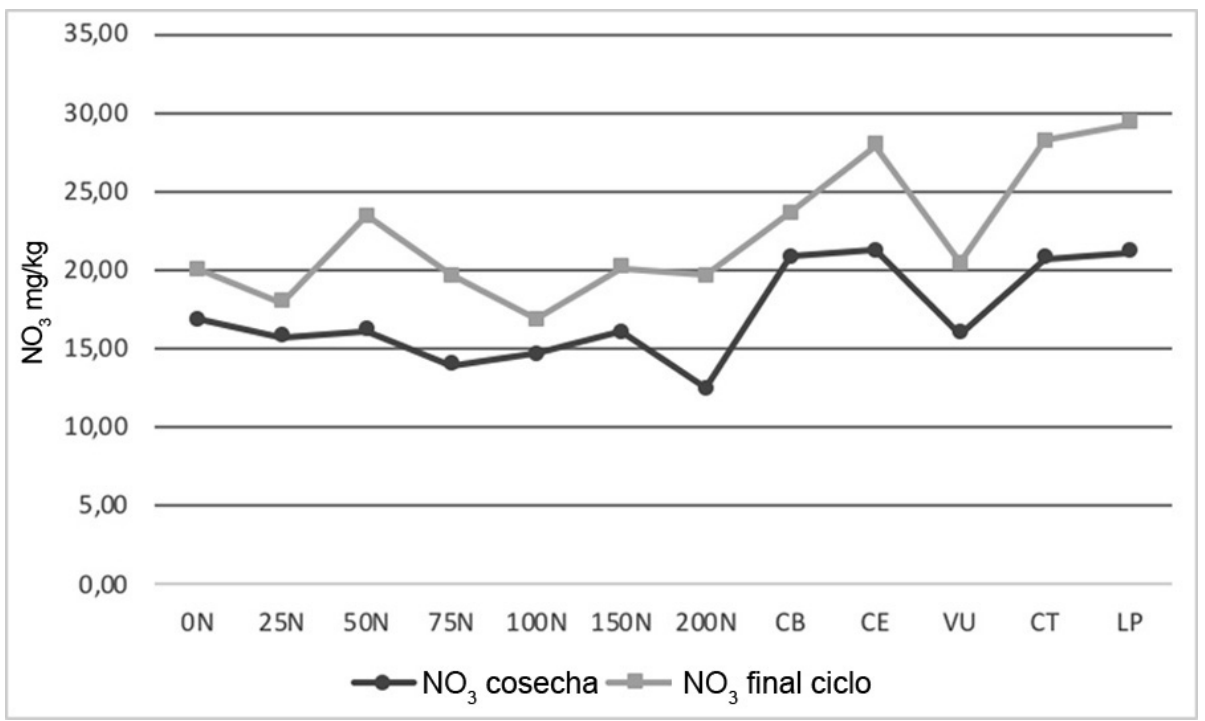

Figura 6. Dinámica de nitrato disponible $(\mathrm{NO} 3)$ en el suelo $(0-20 \mathrm{~cm})$ antes de la incorporación de la leguminosa y al final del ciclo del maíz en la Paz, César, Colombia. 2013-2014.

CB: C. brasiliensis; CE: C. ensiformis; VU: V. unguiculata; CT: C. ternatea; VU: V. unguiculata. LP: L. purpureus.

Figure 6. Available nitrate dynamics (NO3) in the soil $(0-20 \mathrm{~cm})$ before the incorporation of the legume and at the end of the corn cycle in La Paz, Cesar, Colombia. 2013-2014.

C. brasiliensis; CE: C. ensiformis; VU: V. unguiculata; CT: C. ternatea; VU: V. unguiculata. LP: L. purpureus. 


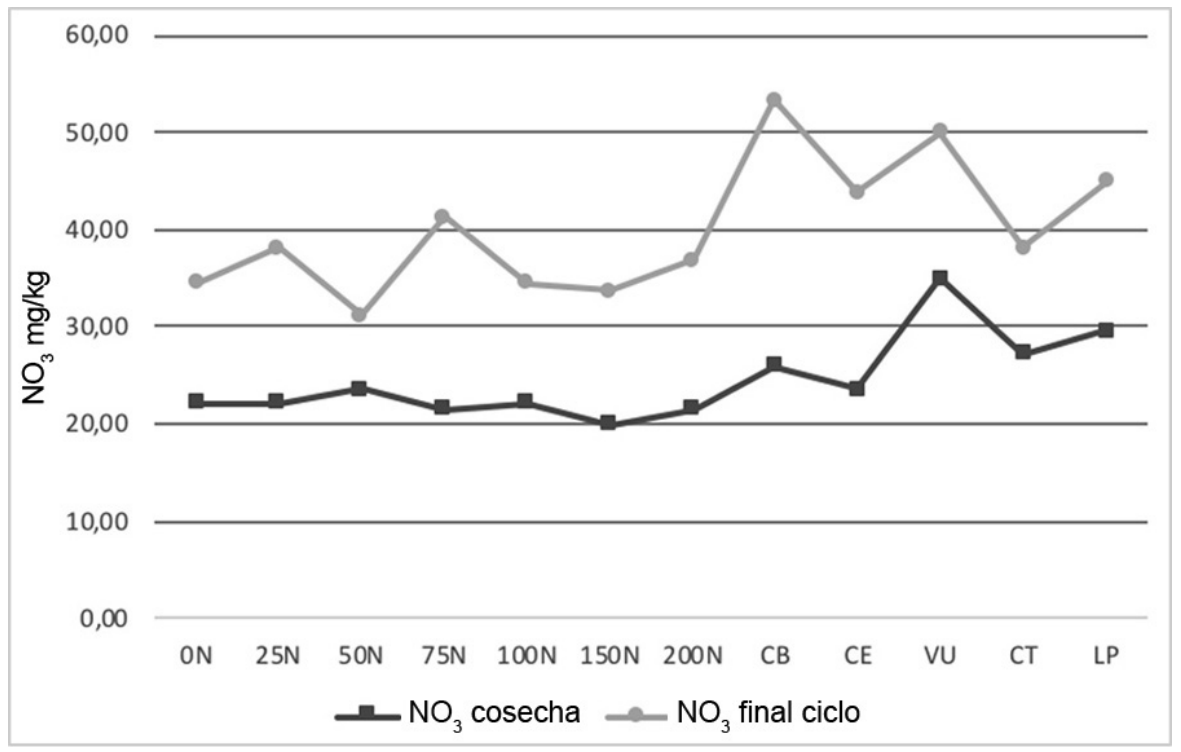

Figura 7. Dinámica de nitrato disponible $(\mathrm{NO} 3)$ en el suelo $(0-20 \mathrm{~cm})$ antes de la incorporación de la leguminosa y al final del ciclo del maíz en Valledupar, César, Colombia. 2013-2014.

CB: C. brasiliensis; CE: C. ensiformis; VU: V. unguiculata; CT: C. ternatea; VU: V. unguiculata. LP: L. purpureus.

Figure 7. Available nitrate dynamics (NO3) in the soil $(0-20 \mathrm{~cm})$ before the incorporation of the legume and at the end of the corn cycle in Valledupar, Cesar, Colombia. 2013-2014.

CB: C. brasiliensis; CE: C. ensiformis; VU: V. unguiculata; CT: C. ternatea; VU: V. unguiculata. LP: L. purpureus.

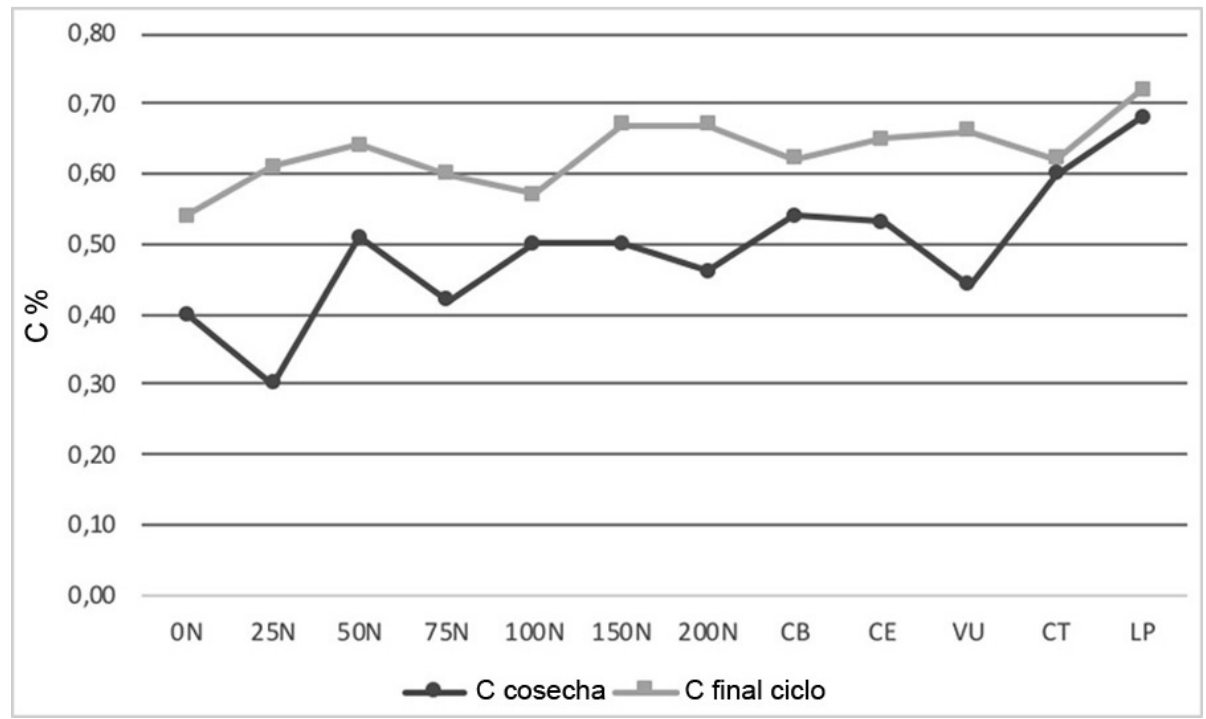

Figura 8. Dinámica de carbono orgánico $(C)$ en el suelo $(0-20 \mathrm{~cm})$ antes de la incorporación de la leguminosa y al final del ciclo del maíz en Agustín Codazzi, César, Colombia. 2013-2014.

CB: C. brasiliensis; CE: C. ensiformis; VU: V. unguiculata; CT: C. ternatea; VU: V. unguiculata. LP: L. purpureus.

Figure 8. Organic carbon dynamics $(\mathrm{C})$ in the soil $(0-20 \mathrm{~cm})$ before the incorporation of the legume and at the end of the corn cycle in Agustin Codazzi, Cesar, Colombia. 2013-2014.

CB: C. brasiliensis; CE: C. ensiformis; VU: V. unguiculata; CT: C. ternatea; VU: V. unguiculata. LP: L. purpureus. 


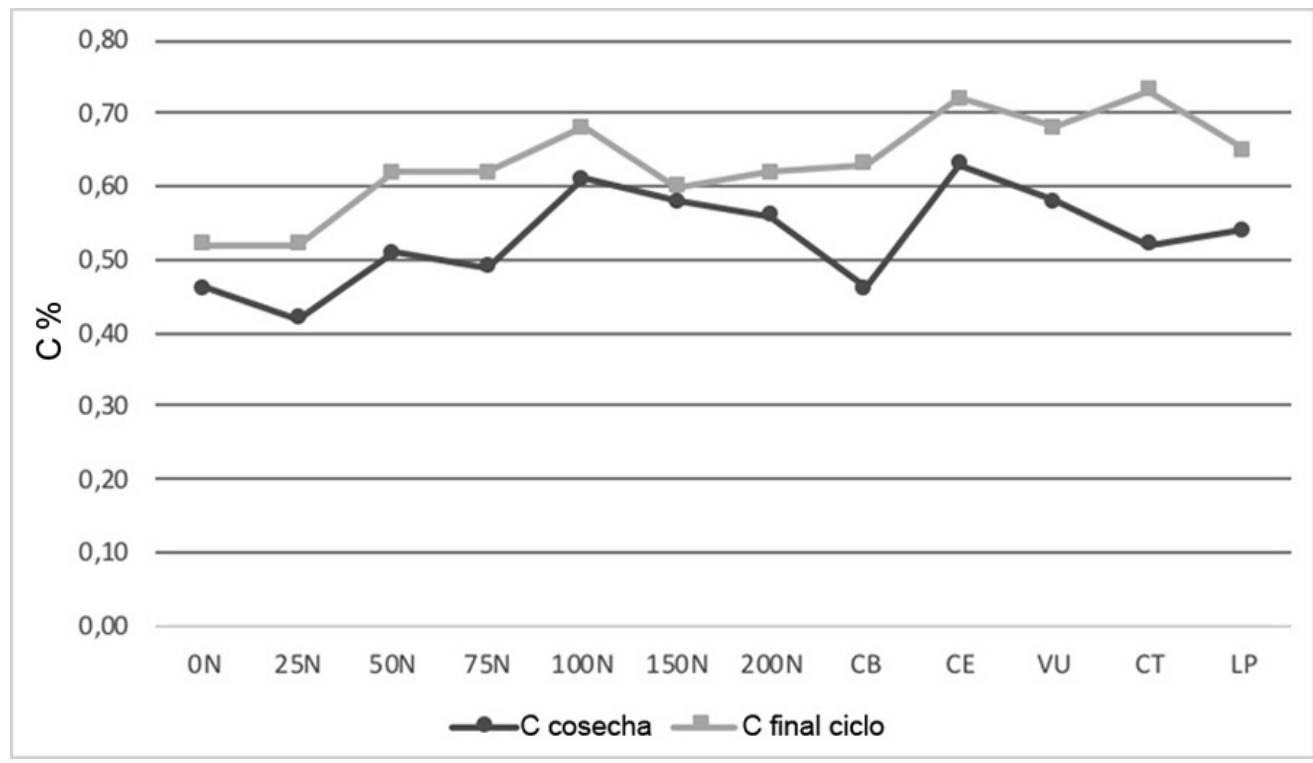

Figura 9. Dinámica de carbono orgánico $(C)$ en el suelo $(0-20 \mathrm{~cm})$ antes de la incorporación de la leguminosa y al final del ciclo del maíz en La Paz, César, Colombia. 2013-2014.

CB: C. brasiliensis; CE: C. ensiformis; VU: V. unguiculata; CT: C. ternatea; VU: V. unguiculata. LP: L. purpureus.

Figure 9. Organic carbon dynamics $(C)$ in the soil $(0-20 \mathrm{~cm})$ before the incorporation of the legume and at the end of the corn cycle in La Paz, Cesar, Colombia. 2013-2014.

CB: C. brasiliensis; CE: C. ensiformis; VU: V. unguiculata; CT: C. ternatea; VU: V. unguiculata. LP: L. purpureus.

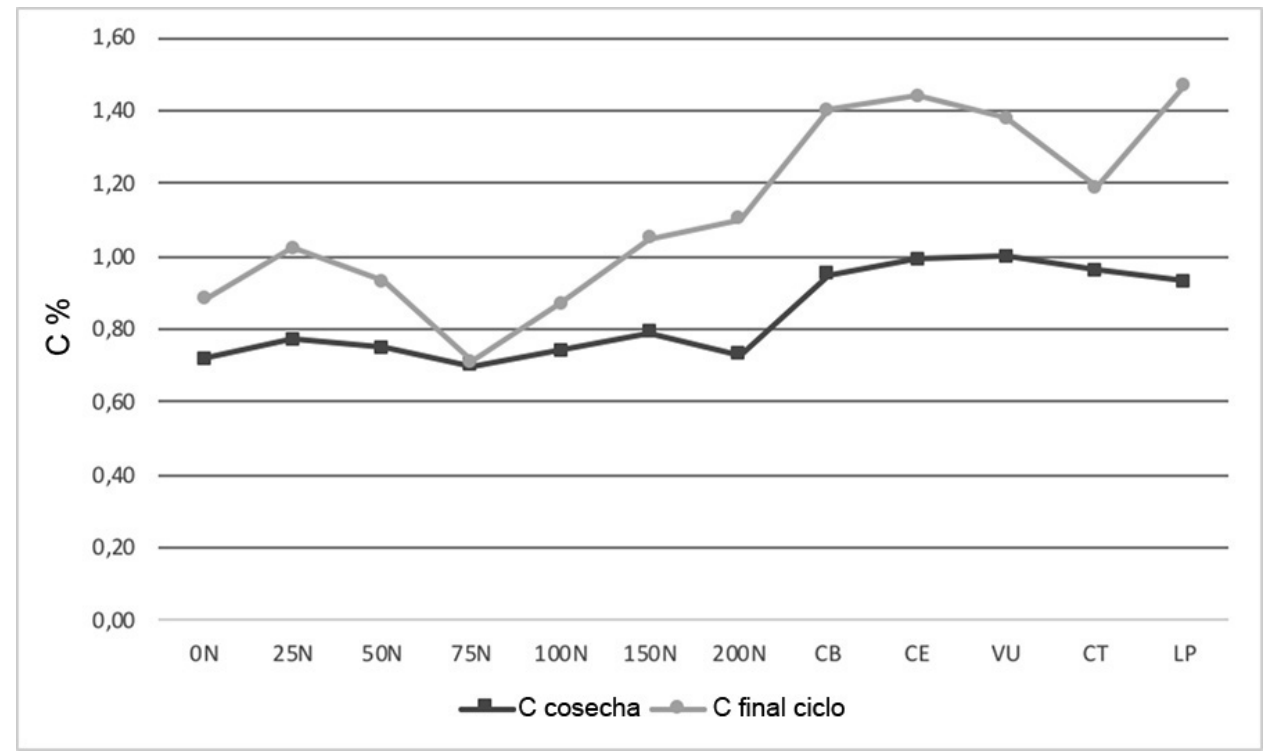

Figura 10. Dinámica de carbono orgánico $(C)$ en el suelo $(0-20 \mathrm{~cm})$ antes de la incorporación de la leguminosa y al final del ciclo del maíz en Valledupar, César, Colombia. 2013-2014.

CB: C. brasiliensis; CE: C. ensiformis; VU: V. unguiculata; CT: C. ternatea; VU: V. unguiculata. LP: L. purpureus.

Figure 10. Organic carbon dynamics $(C)$ in the soil $(0-20 \mathrm{~cm})$ before the incorporation of the legume and at the end of the corn cycle in Valledupar, Cesar, Colombia. 2013-2014.

CB: C. brasiliensis; CE: C. ensiformis; VU: V. unguiculata; CT: C. ternatea; VU: V. unguiculata. LP: L. purpureus. 


\section{Discusión}

La producción de biomasa de $C$. brasiliensis en este estudio fue superior a la reportada por Douxchamps (2010) en Nicaragua (2448 vs. $5357 \mathrm{~kg} \mathrm{MS} / \mathrm{ha}$ ), pero menor a la obtenida (9748 kg MS/ha) en un estudio reportado por Salamanca et al. (2004) en el Valle del Cauca, Colombia. La menor producción observada en este estudio, comparada con otras zonas, puede obedecer a condiciones de suelo y adaptación de los materiales forrajeros empleados.

Los valores de $\mathrm{N}$ equivalente de las leguminosas fueron inferiores a los reportados en Cuyuta, Guatemala, en donde los valores de sustitución de fertilizante fueron de 127 y $158 \mathrm{~kg} \mathrm{~N} /$ ha con Mucuna spp. y C. ensiformis, respectivamente (Pound, 1999).

En Nicaragua con C. ensiformis se observaron valores de N equivalente entre 150 - $200 \mathrm{~kg} / \mathrm{ha}$ (Douxchamps, 2010). En experimentos conducidos en suelos ácidos de los llanos orientales de Colombia se reportó que $C$. ensiformis, V. unguiculata y Crotalaria espectabilis, tuvieron aportes de N que variaron entre 31 y $326 \mathrm{~kg} / \mathrm{ha}$ (Navas y Bernal, 1999). Sin embargo, se debe tener en cuenta que en estos estudios el $\mathrm{N}$ aportado por las leguminosas incorporadas al suelo se calculó a partir de la biomasa de los materiales y de su contenido de $\mathrm{N}$, y no de $\mathrm{N}$ equivalente calculado en función de $\mathrm{N}$ químico aplicado.

En general, los resultados mostraron ventajas comparativas de $C$. ensiformis y $C$. brasiliensis en las tres localidades evaluadas en condiciones del trópico seco del César, tanto en producción de biomasa, humedad del suelo, y aporte de $\mathrm{N}$ al suelo como equivalentes en $\mathrm{kg} \mathrm{N} / \mathrm{ha}$, lo cual coincide con lo reportado en otras zonas de Centro América y África con periodos secos prolongados (Anderson et al., 1997; Keller et al., 2005; Cherr et al., 2006; Maass et al., 2010).

Los contenidos de minerales observados en las leguminosas evaluadas estuvieron dentro de los rangos (N: 1,5 a 3,7\%, P: 0,11 a $0,4 \%, \mathrm{~K}: 1,3$ a 3,5\%, Ca: 0,8 a 1,8\% y Mg: 0,4 a 0,7\%) reportados en otros estudios realizados en el Valle del Cauca, Colombia (Cobo et al., 2002), México, Nicaragua (Pauth-Meza et al., 1997; Vivas-May et al., 2011) y Honduras (Jara-Flores, 1997).

Los resultados de aporte equivalente de $\mathrm{N}$ con la biomasa incorporada de las leguminosas evaluadas y su efecto en producción de maíz en este estudio, están en la misma dirección de otros estudios reportados en la literatura. Sin embargo, los resultados no son comparables, pues en esos estudios se midió grano y no biomasa del cultivo indicador (grano en estado lechoso más follaje). Por ej., Fischler y Wortmann (1999) en el este de Uganda, utilizaron Crotalaria ochroleuca, Mucuna pruriens, Dolichos lablab y C. ensiformis como abonos verdes, y observaron rendimientos de grano de maíz y frijol de un 50 a $60 \%$ más altos en comparación con rendimientos obtenidos sin abonos verdes. En Kenia, luego de la incorporación de M. pruriens C. ensiformis y L. purpureus, se aumentó la producción de grano de maíz de 35 a 100\% en comparación con la aplicación de fertilizante nitrogenado (Mureithi et al., 2003).

Entre las localidades de este estudio, la mayor producción de maíz se obtuvo en Motilonia, seguida de La Paz y Valledupar con la menor $(\mathrm{p}<0,05)$, lo cual se asocia tanto a diferencias en los suelos donde se establecieron los ensayos, como en los niveles de biomasa de leguminosa incorporados, además de cambios en la humedad en el suelo durante el desarrollo de los experimentos.

Los valores de eficiencia de uso de $\mathrm{N}$ fueron superiores al 39\% para las leguminosas, destacando valores mayores a $80 \%$ en V. unguiculata, lo que supera incluso los observados en Cuba (32 a 40\%) con el mismo método de cálculo con abonos verdes de crotalaria, mucuna y sesbania (Martín y Rivera, 2002). En estudios en Venezuela la eficiencia de uso de N químico varió entre 40 y 90\% (Delgado et al., 2001). Se debe tener en cuenta que la eficiencia de uso del $\mathrm{N}$ fijado por leguminosas es normalmente menor que la del $\mathrm{N}$ adicionado como fertilizante químico (Barrios et al., 1996; Chikowo et al., 2006). Sin embargo, la eficiencia de uso del N fijado por una leguminosa usada como abono verde puede variar dependiendo de la sincronización entre el momento de aporte de nutriente y la demanda del cultivo (Seneviratne, 2000; Cobo et al., 2002). 
Para interpretar los valores de $\mathrm{NO}_{3}$ observados en el suelo en el presente estudio, hay que tener en cuenta que al fertilizar con $\mathrm{N}$ químico se genera un proceso de mineralización en el suelo, mediante el cual parte del $\mathrm{N}$ aplicado se volatiliza $(15-20 \%)$ y otra parte se convierte por acción de bacterias (nitrosomas - nitrobacter) en $\mathrm{NO}_{2}-\mathrm{NO}_{3}$. $\mathrm{El}$ $\mathrm{N}$ que la planta no absorbe se pierde por lixiviación o desnitrificación (25 - 30\%).

Las plantas de parcelas fertilizadas con $\mathrm{N}$ químico toman más $\mathrm{N}$ del suelo que las no fertilizadas (efecto priming), por un efecto del fertilizante sobre la mineralización, a través de los procesos fisicoquímicos y microbiológicos del suelo (Ma et al., 1999; Kuzyakov et al., 2000; Martín y Rivera, 2001).

Con la incorporación de leguminosas como abonos verdes, el $\mathrm{N}$ puede estar disponible hasta noventa días después de la incorporación, sobre todo si las leguminosas tienen taninos (Barrios et al., 1996; Seneviratne, 2000). Estudios en Brasil han reportado que, entre el 85 y el $95 \%$ del N proveniente de abonos verdes de mucuna, canavalia y crotalaria en el suelo, fue absorbido por el cultivo de maíz en los primeros veintinueve días después de la germinación, indicando una rápida mineralización (Rivera y Urquiaga, 1995; García et al., 2001; Chikowo et al., 2006).

Valores por encima de $46 \mathrm{mg} / \mathrm{kg}$ de $\mathrm{NO}_{3}$ se consideran altos, implicando riesgo de lixiviación y contaminación de acuíferos (Anken et al., 2004). En el presente estudio se encontraron niveles de $\mathrm{NO}_{3}$ que variaron entre 20 y $41 \mathrm{mg} / \mathrm{kg}$ de $\mathrm{NO}_{3}$ en el suelo después de la cosecha de maíz, en los tratamientos donde se incorporó abonos verdes de leguminosas. Solo para la localidad de Valledupar se observaron valores por encima de $46 \mathrm{mg} / \mathrm{kg}$ en las parcelas de $C$. brasiliensis y $V$. unguiculata. Esto sugiere que una proporción del $\mathrm{N}$ equivalente aportado por la leguminosa al momento de la cosecha del maíz no lo asimiló la planta. La consecuencia de esto es lixiviación de N, lo cual se podría considerar una limitación de los abonos verdes fácilmente degradables en el suelo (García, 1997; Seneviratne, 2000; Mureithi et al., 2003; Beltrán-Morales et al., 2009).

Lo observado en el contenido de C orgánico y la relación C:N, concuerda con lo reportado en otros estudios, donde se encontraron relaciones C:N entre 9 y 25 en leguminosas como C. brasiliensis, L. purpureus, Dolichos lablab y Mucuna pruriens (Cobo et al., 2002; Douxchamps, 2010; Odhiambo, 2010).

Si bien en este estudio se presentó un aumento del C orgánico del suelo asociado con la incorporación de leguminosas como abono verde en las tres localidades, estas se han utilizado en gran parte del mundo con más éxito para incrementar la cantidad de $\mathrm{N}$ asimilable que para aumentar la cantidad de $\mathrm{MO}$ en el suelo, dado que este último proceso lleva más tiempo y requiere de altas cantidades de materia orgánica para mineralizar (Palm y Sanchez, 1991; Seneviratne, 2000; Nziguheba et al., 2005).

La mineralización del $\mathrm{N}$ en el suelo depende en gran medida de la relación C:N del material incorporado. Un material vegetal incorporado al suelo con una relación C:N alta se descompone más lentamente en el suelo que un material con una relación C:N baja (Martín y Rivera, 2002; Nziguheba et al., 2005). Se ha reportado que plantas con relaciones $\mathrm{C}: \mathrm{N}$ mayores a 27 inmovilizan $\mathrm{N}$, mientras que, plantas con una relación C:N menor a 27 mineralizan $\mathrm{N}$, siendo 25 el valor crítico de equilibrio entre inmovilización y mineralización (Myers et al., 1994; Seneviratne et al., 1999; Seneviratne, 2000; Martín y Rivera, 2002).

Las leguminosas se degradan en el suelo en forma rápida cuando su relación de C:N es del orden de 9,0. En el caso de gramíneas la relación C:N es de 20 o más, lo cual determina su degradación lenta en el suelo (Palm y Sanchez, 1991; Barrios et al., 1997; Sakala et al., 2000).

Las leguminosas sin taninos que se descomponen rápidamente, producen una gran cantidad de $\mathrm{CO}_{2}, \mathrm{y}$ consecuentemente, aportan poca $\mathrm{MO}$ a suelo, pero bastante nitrato $\left(\mathrm{NO}_{3}\right)$ que es una forma de $\mathrm{N}$ más asimilable para las plantas. Alternativamente, leguminosas con taninos que tienen baja relación C:N se descomponen lentamente, dejando un buen residuo de $\mathrm{MO}$ y poco $\mathrm{N}$ disponible en el suelo, ya que, el mismo es utilizado por los microorganismos del suelo para descomponer la leguminosa incorporada y en ese proceso se transforma en N orgánico no disponible para cultivos en rotación como maíz (Palm y Sanchez, 1991; Oglesby y Fownes, 1992; Barrios et al., 1997; Seneviratne, 2000). 
Se pudo evidenciar que un aumento en la humedad del suelo pudo resultar en el mejoramiento de características físicas del mismo, como la agregación y porosidad, y mayor número de macroporos, lo cual es conducente a mejores condiciones para la incorporación de MO al suelo por microorganismos (Gupta y Singh, 1981; Latif et al., 1992). Esto a su vez resulta en un aumento del contenido de $\mathrm{N}$ en el suelo, sobre todo si la humedad es mayor al 10\% (Thönnissen et al., 2000; Palm et al., 2001; Hartwig y Ammon, 2002).

\section{Conclusiones}

Las leguminosas $C$. ensiformis, C. brasiliensis y L. purpureus mostraron estar bien adaptadas al trópico seco, tener un alto potencial para aportar $\mathrm{N}$ al suelo como abonos verdes y contribuir a aumentar la producción de cultivos forrajeros para suplementar ganado en la época seca.

En general, se observó en este estudio variación asociada al tipo de suelo y localidad, para las variables asociadas a dinámica de $\mathrm{N}$ en el suelo. En el cual, se destacaron leguminosas como C. ensiformis, C. brasiliensis y L. purpureus por su alta producción de biomasa, tolerancia a la sequía y potencial para aportar $\mathrm{N}$ al suelo como abonos verdes y de contribuir de esa forma a la producción de forraje y grano de maíz.

Con el uso de abonos verdes de C. ensiformis, C. brasiliensis y L. purpureus se evidenciaron aportes equivalentes de $\mathrm{N}$ de 35 a $173 \mathrm{~kg} \mathrm{~N} / \mathrm{ha}$, con lo cual es posible obtener altos rendimiento maíz y reemplazar parcial o completamente la fertilización con $\mathrm{N}$ químico. Lo anterior está asociado al equivalente de $\mathrm{N}$ asociado a la incorporación de leguminosas como fuente para el cultivo agrícola, y con el empleo de leguminosas que permitan una adecuada relación C:N, mantenimiento de la humedad del suelo en la época seca y un adecuado flujo de nutrientes al cultivo posterior, por la mineralización de la materia orgánica de la leguminosa incorporada.

\section{Agradecimientos}

Los autores expresan agradecimiento a la Corporación Colombiana de Investigación Agropecuaria (AGROSAVIA), por financiar la tesis de doctorado donde se desarrolló este estudio.

\section{Literatura citada}

Anderson, S., N. Férreas, S. Gundel, B. Keane, y B. Pound. 1997. Cultivos de cobertura: componentes de sistemas integrados. Presentado en: Taller Regional Latinoamericano. Universidad Autónoma de Yucatán, Mérida, MEX. 3-6 de febrero.

Anken, T., P. Stamp, W. Richner, and U. Walther. 2004. Plant develop $\neg$ ment, nitrogen dynamics and nitrate leaching from ploughed and direct-sown plots. Schr. Eidgen Forsc. Agrar. Land 63:101.

Ayarza, M., E. Amézquita, I. Rao, E. Barrios, M. Rondón, Y. Rubiano, and M. Quintero. 2007. Advances in improving agricultural profitability and overcoming land degradation in Savanna and Hillside agroecosystems of tropical America. In: A. Bationo et al., editors, Advances in integrated soil fertility management in sub-Saharan Africa: challenges and opportunities. Springer, Dordrecht, HOL. p. 209-229.

Barbieri, P.A., H.E. Echeverría, H.R. Saínz-Rozas, y M. Maringolo. 2010. Fertilización de maíz con urea de liberación lenta: pérdida por volatilización y eficiencia de uso de nitrógeno. Cienc. Suelo 28(1):57-66.

Barrios, E., R.J. Buresh, and J.I. Sprent. 1996. Nitrogen mineralization in density fractions of soil organic matter from maize and legume cropping systems. Soil Biol. Biochem. 28:1459-1465. doi: 10.1016/S0038-0717(96)00155-1 
Barrios, E., F. Kwesiga, R.J. Buresh, and I. Sprent. 1997. Light fraction soil organic matter and available nitrogen following trees and maize. Soil Sci. Soc. Am. J. 61:826-831.

Beltrán-Morales, F.A., J.L. García-Hernández, F.H. Ruiz-Espinoza, L. Fenech-Larios, B. Murillo-Amador, A. PalaciosEspinoza, and E. Troyo-Diéguez. 2009. Nutrimental potential of red dolichos, brown dolichos and cowpea for green manure produced under three tillage systems. Trop. Subtrop. Agroecosyst. 10:487-495.

Biederbeck, V.O., C.A. Campbell, V. Rasiah, R.P. Zentner, and G. Wen. 1998. Soil quality attributes as influenced by annual legumes used as green manure. Soil Biol. Biochem. 30:1177-1185. doi:10.1016/S0028-0717(97)00150-8

Boschini, C., y J. Elizondo. 2004. Rendimiento de forraje de dos materiales genéticos de maíz sembrados a diferentes distancias de siembra. Rev. Agric. Trop. 34:87-92.

Burle, M.L., A.R. Suhet, J. Pereira, D.V.S. Resck, J.R.K. Peres, M.S. Cravo, and D.J. Lathwell. 1992. Legume green manures. Dry season survival and the effect on succeeding maize crops. Bull. 92-04. Soil Managment Collaborative Research Support Program, Raleigh, NC, USA.

Carvalho, M.A., R. Soratto, M. Athayde, O. Arf, e M. de-Sá. 2004. Produtividade do milho em sucessão a adubos verdes no sistema de plantio direto e convencional. Pesqui. Agropecu. Bras. 39:47-53. doi:10.1590/S0100-204X2004000100007

Castro, R.E. 2016. Utilización de leguminosas forrajeras como abonos verdes para la producción de cultivos forrajeros y leche en ganaderías doble propósito en el trópico seco. Tesis Dr., Universidad Nacional de Colombia, Bogotá, COL.

Castro, R.E., A. Sierra, J.E. Mojica, J.E. Carulla, y C.E. Lascano. 2017. Efecto de especies y manejo de abonos verdes de leguminosas en la producción y calidad de un cultivo forrajero utilizado en sistemas ganaderos del trópico seco. Arch. Zootec. 66:99-106. doi:10.21071/azz.v66i253.2131

Castro-Rincón, E., A.M. Sierra-Alarcón, J.E. Mojica-Rodríguez, J. Carulla-Fornaguera, y C. Lascano-Aguilar. 2016. Uso múltiple de leguminosas como abono verde, en rotación con maíz, y heno, para producción de leche. Corpoica Cienc. Tecnol. Agropecu. 17:17-29.doi:10.21930/rcta.vol17_num1_art:456

Cherr, C.M., J.M.S. Scholberg, and R. McSorley. 2006. Green manure approaches to crop production: A synthesis. Agron. J. 98:302-319. doi:10.2134/agronj2005.0035

Chikowo, R., P. Mapfumo, P.A. Leffelaar, and K.E. Giller. 2006. Integrating legumes to improve N cycling on smallholder farms in sub-humid Zimbabwe: Resource quality, biophysical and environmental limitations. Nutr. Cycl. Agroecosyst. 76:219231. doi: $10.1007 / \mathrm{s} 10705-005-2651-y$

Cobo, J.G., E. Barrios, D.C.L. Kass, and R.J. Thomas. 2002. Decomposition and nutrient release by green manures in a tropical hillside agroecosystem. Plant Soil 240:331-342. doi:10.1023/A:10157232

Delgado, R., R. Ramírez, y S. Urquiaga. 2001. Colocación del nitrógeno en el suelo y la eficiencia de uso por el maíz. Agron. Trop. 51:337-350.

Douxchamps, S. 2010. Integration of Canavalia brasiliensis into the crop-livestock system of the Nicaraguan hillsides: environmental adaptation and nitrogen dynamics. Thesis DrSc., Swiss Federal Institute of Tecnology Zurich, Zürich,SUI.

Douxchamps, S., F.L. Humbert, R. van-der-Hoek, M. Mena, S.M. Bernasconi, A. Schmidt, I. Rao, E. Frossard, and A. Oberson. 2010. Nitrogen balances in farmers fields under alternative uses of a cover crop legume: A case study from Nicaragua. Nutr. Cycl. Agroecosyst. 88:447-462. doi:10.1007/s10705-010-9368-2

Douxchamps, S., I.M. Rao, M. Peters, R. va-der-Hoek, A. Schmidt, S. Martens, J. Polania, M. Mena, C.R. Binder, R. Schöll, M. Quintero, M. Kreuzer, E. Frossard, and A. Oberson. 2014. Farm-scale tradeoffs between legume use as forage versus green manure: The case of Canavalia brasiliensis. Agroecol. Sustain. Food Syst. 38:25-45. doi:10.1080/21683565.2013 .828667 
Fischler, M., and C.S. Wortmann. 1999. Green manures for maize-bean systems in eastern Uganda: Agronomic performance and farmers' perceptions. Agrofor Syst. 47:123-138. doi:10.1023/A:1006234523163

García, M. 1997. Contribución al estudio y utilización de los abonos verdes en cultivos económicos desarrollados sobre un suelo Ferralítico Rojo de La Habana. Tesis Dr., INCA, La Habana, CUB.

García, M., E. Treto, y M. Álvarez. 2001. Comportamiento de diferentes especies de plantas para ser utilizadas como abonos verdes en las condiciones de Cuba. Cultiv. Trop. 22(4):11-16.

Gupta, S.R., and J.S. Singh. 1981. The effect of plant species, weather variables and chemical composition of plant material on decomposition in a tropical grassland. Plant Soil 59:99-117. doi:10.1007/BF02183596

Hartwig, N.L., and H.U. Ammon. 2002. Cover crops and living mulches. Weed Sci. 50:688-699. doi:10.1614/00431745(2002)050[0688:AIACCA]2.0.CO;2

Jara-Flores, A.F. 1997. Evaluación del aporte de tres leguminosas (Canavalia ensiformis, Mucuna deeringianum, Dolichos lablab) usadas como abono verde sobre la recuperación de suelos de ladera degradados. Tesis Lic., Escuela Agrícola Panamericana, Zamorano, HON.

Keller, G.B., H. Mndiga, and B.L. Maass. 2005. Diversity and genetic erosion of traditional vegetables in Tanzania from the farmer's point of view. Plant Genet. Resour. 3:400-413. doi:10.1079/PGR200594

Krom, M.D. 1980. Spectrophotometric determination of ammonia: a study of a modified Berthelot reaction using salicylate and dichloroisocyanurate. Analyst 105:305-316. doi:10.1039/AN9800500305

Kuzyakov, Y., J.K. Friedel, and K. Stahr. 2000. Review of mechanisms and quantification of priming effects. Soil Biol. Biochem. 32:1485-1498. doi:10.1016/S0038-0717(00)00084-5

Latif, M.A., G.R. Mehuys, A.F. Mackenzie, I. Alli, and M.A. Faris. 1992. Effects of legumes on soil physical quality in a maize crop. Plant Soil 140:15-23. doi:10.1007/BF00012802

Ma, B.L., L.M. Dwyer, and E.G. Gregorich. 1999. Soil nitrogen amendment effects on seasonal nitrogen mineralization and nitrogen cycling in maize production. Agron. J. 91:1003-1009. doi:10.2134/agronj1999.916003x

Maass, B.L., M.R. Knox, S.C. Venkatesha, T.T. Angessa, S. Ramme, and B.C. Pengelly. 2010. Lablab purpureus A crop lost for Africa? Trop. Plant Biol. 3:123-135. doi:10.1007/s12042-010-9046-1

Martín, G.M., y R. Rivera. 2001. Mineralización del nitrógeno incorporado con los abonos verdes y su participación en la nutrición de cultivos de importancia económica. Cultivos Trop. 22(3):89-96.

Martín, G.M., y R. Rivera. 2002. Participación del nitrógeno de los abonos verdes en la nutrición nitrogenada del maíz (Zea mays L.) cultivados sobre suelos ferralítico rojo. Cultivos Trop. 23(3):91-96.

Muraoka, T., E. Ambrosano, F. Zapata, N. Bortoletto, A.L.M. Martins, P.C.O. Trivelin, A.E. Boaretto, y W.B. Scivittaro. 2002. Eficiencia de abonos verdes (crotalaria y mucuna) y urea, aplicados solos o juntamente, como fuentes de n para el cultivo de arroz. Terra Latinoam. 20(1):17-23.

Mureithi, J.G., C.K.K. Gachene, and J. Ojiem. 2003. The role of green manure legumes in smallholder farming systems in Kenya: the legume research network project. Trop. Subtrop. Ecosyst. 1:57-70.

Myers, R., C. Palm, E. Cuevas, I. Gunatilleke, and M. Brossard. 1994. The synchronisation of nutrient mineralisation and plant nutrient demand. In: P. Woomer, and M. Swift, editors, The biological management of tropical soil fertility. Wiley, Chichester, GBR. p. 81-116.

Navas G., y J. Bernal. 1999. Caracterización de leguminosas como abono verde para los sistemas de producción del Piedemonte Llanero y Altillanura Colombiana. Boletín Técnico No. 16. Corpoica, Villavicencio, COL. 
Nelson, D.W., and L. Sommers. 1982. Total carbon, organic carbon, and organic matter. In: A.L. Page et al., editors, Methods of soil analysis, Part 2. Chemical and microbiological properties-Agronomy Monograph No. 9. $2^{\text {nd }}$ ed. ASA Inc., and SSSA Inc., Madison, WI, USA. p. 539-579.

Nziguheba, G., R. Merckx, and C.A. Palm. 2005. Carbon and nitrogen dynamics in a phosphorus-deficient soil amended with organic residues and fertilizers in western Kenya. Biol. Fertil. Soils 41:240-248. doi:10.1007/s00374-005-0832-0

Odhiambo, J.J.O. 2010. Decomposition and nitrogen release by green manure legume residues in different soil types. African J. Agric. Res. 5:90-96. doi:10.5897/AJAR09.489

Oglesby, K.A., and J.H. Fownes. 1992. Effect of chemical composition on nitrogen mineralization from green manures of seven tropical leguminous trees. Plant Soil 143:127-132. doi:10.1007/BF00009137

Palm, C.A., C.N. Gachengo, R.J. Delve, G. Cadisch, and K.E. Giller. 2001. Organic inputs for soil fertility management in tropical agro ecosystems: application of an organic resource database. Agric. Ecosyst. Environ. 83:27-42. doi:10.1016/ S0167-8809(00)00267-X

Palm, C.A., and P.A. Sanchez. 1991. Nitrogen release from the leaves of some tropical legumes as affected by their lignin and polyphenolic contents. Soil Biol. Biochem. 23:83-88. doi:10.1016/0038-0717(91)90166-H

Pauth-Meza, N.J., L.H. Olivas-Lira, y L.H. Palma-Marín. 1997. Caracterización y evaluación preliminar de germoplasma de leguminosas nativas con potencial forrajero en Jalapa y Estelí, Nicaragua. Tesis Tec. Agr., Escuela de Agricultura y Ganadería de Estelí Francisco Luis Espinoza Pineda, Estelí, NIC.

Phiri, S., E. Barrios, I.M. Rao, and B.R. Singh. 2001. Changes in soil organic matter and phosphorus fractions under planted fallows and a crop rotation system on a Colombian volcanic-ash soil. Plant Soil 231:211-223. doi:10.1023/A:1010310300067

Pound, B. 1999. Cultivos de cobertura para la agricultura sostenible en América Latina. En: FAO, editor, Agroforestería para la producción animal en Latinoamérica. FAO, Roma, ITA. p. 143-168.

Rivera, R., y S. Urquiaga. 1995. Mineralización y participación del nitrógeno de tres especies de abonos verdes en la nutrición nitrogenada del maíz. Presentado en: Encuentro nacional de agricultura orgánica. ACAO, La Habana, CUB. 17-19 de mayo.

Sakala, W.D., G. Cadisch, and K.E. Giller. 2000. Interactions between residues of maize and pigeonpea and mineral N fertilizers during decomposition and N mineralization. Soil Biol. Biochem. 32:679-688. doi:10.1016/S0038-0717(99)00204-7

Salamanca, W.F., C.R. Bonilla, y M.S. Sánchez. 2004. Evaluación de seis abonos verdes en un vertisol ústico en condiciones del Valle del Cauca. Acta Agron. 53(3):55-60.

Seneviratne, G. 2000. Litter quality and nitrogen release in tropical agriculture: a synthesis. Biol. Fertil. Soils 31:60-64. doi: $10.1007 / \mathrm{s} 003740050624$

Seneviratne, G., L.H.J. Van-Holm, L.J.A. Balachandra, and S.A. Kulasooriya. 1999. Differential effects of soil properties on leaf nitrogen release. Biol. Fertil. Soils 28:238-243. doi:10.1007/s003740050488

Smyth, J., M.A. Ayarza, L. Brizuela, and P.P. Orozco. 2004. Testing diagnosis of the NuMaSS expert system for N and P applications in corn-based systems. In: CIAT, editor, Integrated soil fertility management in the tropics. TSBF annual report. CIAT, Cali, COL. p. 123-125.

Soto O., P., E. Jahn B., y S. Arredondo S. 2004. Mejoramiento del porcentaje de proteína en maiz para ensilaje con el aumento y parcialización de la fertilización nitrogenada. Agric. Téc. 64:156-162. doi:10.4067/S0365-28072004000200004

Tan, Z.X., R. Lal, and K.D. Wiebe. 2005. Global soil nutrient depletion and yield reduction. J. Sustain. Agric. 26:123-146. doi:10.1300/J064v26n01_10 
Thönnissen, C., D.J. Midmore, J.K. Ladha, D.C. Olk, and U. Schmidhalter. 2000. Legume decomposition and nitrogen release when applied as green manures to tropical vegetable production systems. Agron. J. 92:253-260. doi: 10.2134/ agronj2000.922253x

Thurston, H.D. 1992. Sustainable practices for plant disease management in traditional farming systems. CRC Press, Boca Raton, FL, USA.

Toledo, J.M., y R. Shultze-Kraft. 1982. Metodología para la evaluación agronómica de pastos tropicales. In: J.M. Toledo, editor, Manual para la evaluación agronómica: Red Internacional de Evaluación de Pastos Tropicales. CIAT, Cali, COL. p. 91-110.

Valero, J.A., M. Maturano, A. Ramírez, J.M. Martín-Benito, and J.F. Álvarez. 2005. Growth and nitrogen use efficiency of irrigated maize in a semiarid region as affected by nitrogen fertilization. Spanish J. Agric. Res. 3:134-144. doi:10.5424/ sjar/2005031-133

Vivas-May, E.F., J.G. Rosado-Rubio, A.F. Castellanos-Ruelas, M. Heredia-y-Aguilar, y E.J. Cabrera-Torres. 2011. Contenido mineral de forrajes en predios de ovinocultores del estado de Yucatán. Rev. Mex. Cienc. Pecu. 2:465-475. 